\title{
Aerodynamics of smooth and rough square-section prisms at incidence in very high Reynolds-number cross-flows
}

\author{
Nils Paul van Hinsberg ${ }^{1}$ \\ Received: 2 August 2020 / Revised: 5 January 2021 / Accepted: 9 January 2021 / Published online: 1 March 2021 \\ (c) The Author(s) 2021
}

\begin{abstract}
The aerodynamics of smooth and slightly rough prisms with square cross-sections and sharp edges is investigated through wind tunnel experiments. Mean and fluctuating forces, the mean pitch moment, Strouhal numbers, the mean surface pressures and the mean wake profiles in the mid-span cross-section of the prism are recorded simultaneously for Reynolds numbers between $1 \times 10^{5} \leq \operatorname{Re}_{D} \leq 1 \times 10^{7}$. For the smooth prism with $k_{s} / D=4 \times 10^{-5}$, tests were performed at three angles of incidence, i.e. $\alpha=0^{\circ},-22.5^{\circ}$ and $-45^{\circ}$, whereas only both "symmetric" angles were studied for its slightly rough counterpart with $k_{s} / D$ $=1 \times 10^{-3}$. First-time experimental proof is given that, within the accuracy of the data, no significant variation with Reynolds number occurs for all mean and fluctuating aerodynamic coefficients of smooth square prisms up to Reynolds numbers as high as $\mathcal{O}\left(10^{7}\right)$. This Reynolds-number independent behaviour applies to the Strouhal number and the wake profile as well. In contrast to what is known from square prisms with rounded edges and circular cylinders, an increase in surface roughness height by a factor 25 on the current sharp-edged square prism does not lead to any notable effects on the surface boundary layer and thus on the prism's aerodynamics. For both prisms, distinct changes in the aerostatics between the various angles of incidence are seen to take place though.
\end{abstract}

Nils Paul van Hinsberg

nils.vanhinsberg@dlr.de

1 Institute of Aeroelasticity, German Aerospace Center,

Göttingen, Germany 


\section{Graphic abstract}
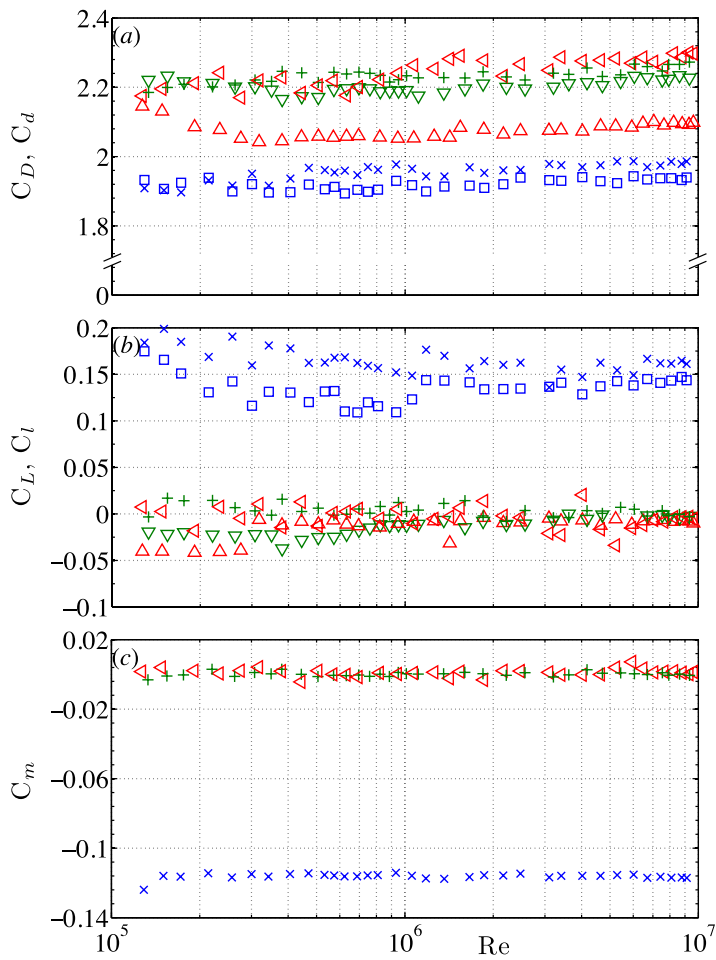

\section{Introduction}

The aerodynamic and aeroelastic behaviour of cylindrical structures with bluff cross-sections has received considerable attention in many different research fields like aerospace (Gatto and Graham 2017; Povoa et al. 2018), civil (Demartino et al. 2015; Chen et al. 2017; Yu et al. 2017; Helgedagsrud et al. 2019) and mechanical engineering (Caliskan 2014; Mangrulkar et al. 2019), as well as in marine sciences (Forrest et al. 2016; Shukla et al. 2019) and wind engineering (Bagbanci et al. 2012; Hall et al. 2014). The main motivation of the many experimental, numerical and analytical studies in the field of bluff body aerodynamics lies in the desire to finally being able to capture the highly complex relation between object shape, the flow behaviour around this type of bluff bodies and the resultant fluid-structure interactions into a robust theoretical model. In this context, the studied fluid flow around generic circular and prismatic structures has to be seen as an idealised representation of realistic applications. Through systematic scientific studies of the flow around single structural elements, the underlying valuable physics of the time-dependent aero- or hydrodynamic behaviour of those elements can be obtained, like the drag crisis and associated bistable state within the critical
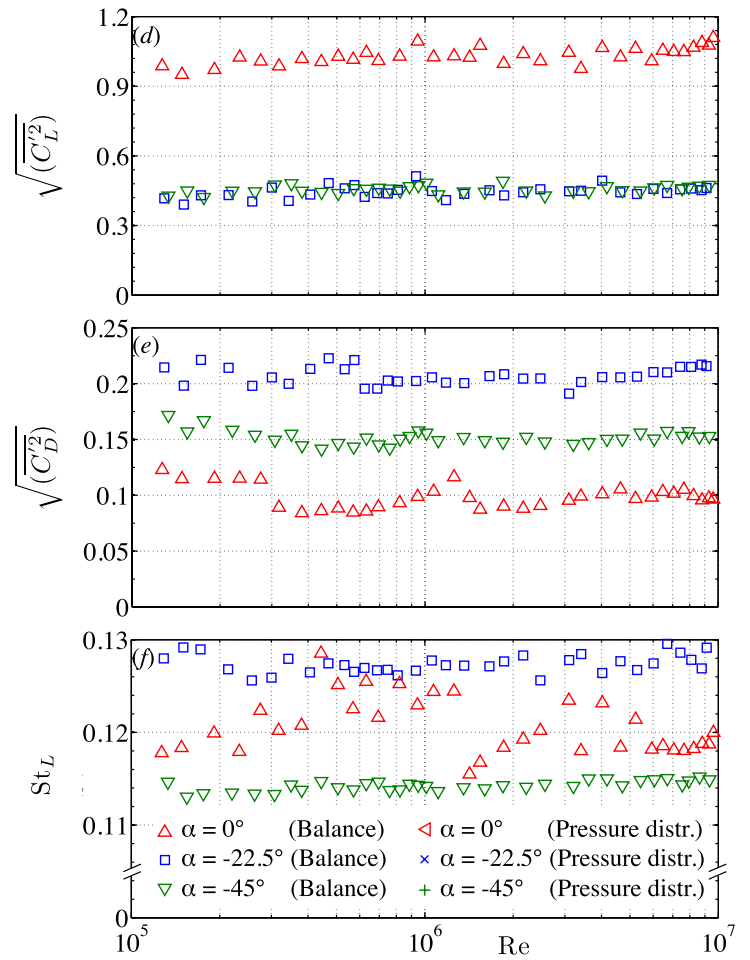

Reynolds-number range of circular cylinders (e.g. Schewe 1983; Desai et al. 2020 and references therein), dry galloping of inclined cables (Virlogeux 2005; Nikitas and Macdonald 2015) and vortex-induced vibration (VIV) for circular cylinders (Païdoussis et al. 2011), as well as transverse and rotational galloping for prismatic structures (e.g. Amandolèse and Hémon 2010; Païdoussis et al. 2011). Thorough knowledge on these aspects is required when studying the much more complicated flow behaviour of multi-cylinder and multi-prism structures, like offshore platforms and floating wind turbines (Gonçalves et al. 2012, 2013; Cheng et al. 2017), industrial heat exchangers (Mangrulkar et al. 2019), high-rise districts (Irwin et al. 2013; Zu and Lam 2018) and cable-stayed bridges (Tanaka 2003; Virlogeux 2005; Nikitas and Macdonald 2015).

\subsection{Aerodynamics of prisms with square cross-sections}

Two-dimensional prisms with square (or rectangular) crosssections and sharp edges are positioned at one outer side of the wide spectrum of possible cross-sectional shapes. Whereas the flow structures around and the aerodynamic loading on 2D circular cylinders in cross-flow both possess 
a strong Reynolds-number dependency over a very broad range of Reynolds numbers (e.g. Roshko 1961; Achenbach 1968; Bearman 1969; Farell and Blessmann 1983; Schewe 1983; Williamson 1996; Zdravkovich 1997), the influence of the Reynolds number on the aerodynamics of sharp-edged square-section prisms at $0^{\circ}$ angle of incidence is limited to relatively low Reynolds numbers up to $R e_{D} \approx 1 \times 10^{3}$ [see, for example, Bai and Alam (2018) and references therein]. The shear layer transition II regime, present at $R e_{D}>1 \times$ $10^{3}$, has been extensively investigated in the last couple of decades, in particular for prism flows at relatively high Reynolds numbers of $\mathcal{O}\left(10^{4}-10^{5}\right)$. In this flow regime, the separation of the surface boundary layer takes place at both upstream edges, and the transition from laminar to turbulent flow is located firmly at both free shear layers above the side surfaces of the prism. The various flow parameters therefore all reach approximately constant values (e.g. $C_{D}=2.21, W^{\star}$ $=1.15$ and $S t=0.13$ ), independent of a further increase in Reynolds number (Okajima 1982; Norberg 1993; Sohankar 2006; Brun et al. 2008a; Minguez et al. 2011; Bai and Alam 2018).

Detailed measurements with the laser Doppler velocimetry technique (LDV) were taken by Brun et al. (2008a) and Minguez et al. (2011) at $R e_{D}=2 \times 10^{4}-3 \times 10^{5}$ and $R e_{D}$ $=1.4 \times 10^{4}$, respectively, to analyse the development of the small-scale Kelvin-Helmholtz-type vortical structures in the free shear layers above the side surfaces of the prism at $\alpha$ $=0^{\circ}$, as well as the large-scale von Kármán vortex street in its near wake. Fluctuating and time- and phase-averaged characteristics of both the flow structures and their typical length and velocity scales in the base region, as well as in the near and far wake of a sharp-edged square prism in crossflow, were obtained for a wide range of Reynolds numbers by Durao et al. (1988) and Lyn et al. (1995) using the LDV technique in a water tunnel, by Saha et al. (2000), Hacişevki and Teimourian (2015), and Bahrami and Hacişevki (2019) using hot-wire anemometry (HWA), by $\mathrm{Hu}$ et al. (2006) using particle image velocimetry (PIV) and by Bai and Alam (2018) through measurements using a combination of the last two measurement techniques. Based on the detailed experiments by Durao et al. (1988) and Lyn et al. (1995), a large amount of research effort has been put into numerical simulations of the small-scale and large-scale flow structures at the specific Reynolds number of $R e_{D}=2.2 \times 10^{4}$, both by high-order LES approaches (Minguez et al. 2011; Cao and Tamura 2016), by direct numerical simulations (DNS) (Saha et al. 2001; Trias et al. 2015) and by Reynolds-averaged Navier-Stokes simulations (RANS) and hybrid LES/RANS simulations (Ke 2019).

The characteristic trends of the mean and fluctuating lift and drag coefficients, the Strouhal and the base pressure coefficient with angle of incidence of sharp-edged square prisms in a wide range of Reynolds numbers have also been the subject of many studies, e.g. Norberg (1993), Luo et al. (1994), Lyn et al. (1995), Chen and Liu (1999), Tamura and Miyagi (1999), Sohankar (2006), van Oudheusden et al. (2007), Huang et al. (2010), Huang and Lin (2011), Yen and Yang (2011) and Carassale et al. (2014). Early measurements of the mean drag coefficient and the Strouhal number as functions of the angle of incidence by Lee (1975), Rockwell (1977), Obasaju (1983), Knisely (1990), and Chen and Liu (1999) showed that a minimum drag coefficient in combination with a sharp rise of the Strouhal number to its maximum value occurs around the critical angle of incidence of $\alpha=13^{\circ}-17^{\circ}$. Both phenomena are related to a shear layer reattachment onto the downstream portion of the windward-directed side surface. The strong pressure recovery on this side surface and the resultant asymmetric flow field around the prismatic body result in a deflection of the shear layer and the formation of a weaker vortex in the base region, hence a higher base pressure and thus a lower drag coefficient, as well as a higher vortex shedding frequency. These trends of the drag coefficient and the Strouhal number were later on confirmed by Dutta et al. (2003) through a wind tunnel study on the two-component time-varying velocity in the wake of prisms at angles of incidence between $0^{\circ}$ $-60^{\circ}$ and Reynolds numbers of 1340,4990 and 9980 . They concluded that the reasons for these trends at $\alpha>0^{\circ}$ are a downstream movement of the separation points and a widening of the wake in combination with a reduction of the vortex formation length.

Igarashi (1984) used the surface oil-flow and smoke visualisation techniques to study the flow pattern around a square prism at angles of incidence of $0^{\circ}-45^{\circ}$ and $R e_{D}=3.85 \times$ $10^{3}-7.7 \times 10^{4}$. Based on the smoke images and the trends of multiple aerodynamic parameters with increasing angle of incidence, he identified three main flow regimes: the perfect separated flow regime for $\alpha<15^{\circ}$, in which the forward separation is pinpointed at the windward sharp edges of the prism, the reattachment flow regime for $\alpha=15^{\circ}-35^{\circ}$ with a separation bubble on the windward-directed side surface of the prism, thereby forming a separation bubble, and the wedge flow regime with an attached flow on both upstream-directed surfaces for $\alpha>35^{\circ}$. The different features of the topological flow patterns of a sharp-edged square prism at angles of incidences from $0^{\circ}$ to $45^{\circ}$ were later on investigated experimentally by Huang et al. (2010) and Yen and Yang (2011) at $R e_{D}$ $=1.3 \times 10^{4}-9.4 \times 10^{4}$ and $R e_{D}=4.0 \times 10^{3}-3.6 \times 10^{4}$, respectively, to solve the physical mechanism occurring in the vicinity of the critical angle of incidence and the associated effects on the wake properties. van Oudheusden et al. (2005) and Ozgoren (2006) used PIV to resolve the instantaneous, phased- and time-averaged vorticity, the vortex-formation lengths and the two-dimensional distributions of the mean and fluctuating flow 
velocities in both the base region and the near wake of a square prism at various angles of incidence.

\subsection{Objectives of the present study}

The previous overview has shown that extensive research on the aerostatics of smooth prisms with square cross-sections and sharp edges with angle of incidence has been conducted at Reynolds numbers up to $\mathcal{O}\left(10^{5}\right)$. Independent of their angle of incidence, the presence of the sharp edges imposes the laminar or turbulent boundary layer detachment at specific surface locations, thereby making the square prisms in the shear layer transition II regime practically insensitive to a variation in Reynolds number. Although no experimental proof has been given until now, it is stated in ESDU (1971), that for Reynolds numbers beyond $10^{5}$ no significant effect on the force coefficients is therefore to be expected as well.

Studies on smooth and rough circular cylinders and square prisms with rounded edges in steady cross-flows at high Reynolds numbers have shown the large impact of surface irregularities, like spanwise roughness stripes, uniformly distributed dimples, grooves, solid particles or large sheets of glass or sand paper, on the dynamic loading on and the flow topology around these kinds of objects (van Hinsberg 2015; van Hinsberg et al. 2018 and the many references therein). Besides higher drag forces, the change in the behaviour of the surface boundary layer with increasing surface roughness induces an increase in both the vortex strength and its spanwise coherence, thus giving rise to even higher periodic lift forces, and a reduction of the vortex shedding frequency. The question as to whether a similar behaviour may exist for sharp-edged square prisms remains so far unanswered, since neither experimental nor numerical data on the aerodynamic behaviour of rough sharp-edged square prisms is available up to date.

With the twofold purpose of investigating the generally presumed Reynolds-number independency of smooth sharpedged square prisms for $R e_{D} \geq \mathcal{O}\left(10^{5}\right)$ and the basic aerostatics of rough sharp-edged square prisms, a wind tunnel study has been performed on both bluff bodies. The global mean and fluctuating lift and drag forces, the 2D mean pitch moment, the Strouhal number, as well as the mean surface pressures and the mean wake profile in the mid-span crosssection have been measured for Reynolds numbers between $10^{5}$ and $10^{7}$. Two surface roughness heights, i.e. $k_{s} / D=4 \times$ $10^{-5}$ and $1 \times 10^{-3}$, were selected, and three angles of incidence, $\alpha=0^{\circ},-22.5^{\circ}$ and $-45^{\circ}$, were investigated.

\section{Experimental arrangement}

The wind tunnel test set-up and the instrumentation were similar to those described in detail in van Hinsberg et al. $(2017,2018)$. The Göttingen-type high-pressure wind tunnel of the German-Dutch wind tunnels (DNW) has a test section of $0.6 \times 0.6 \times 1 \mathrm{~m}^{3}$. The air inside the entire wind tunnel tube can be pressurised up to $10 \mathrm{MPa}$ to achieve maximum Reynolds numbers, based on the width of the prism, of $10^{7}$ in a low subsonic flow of $U_{\infty, \max }=35 \mathrm{~m} / \mathrm{s}$. The free-stream turbulence intensity (T.I.) in the test section shows a slight increase with increasing Reynolds number from T.I. = $0.15 \%$ up to a maximum value of about T.I. $=0.8 \%$, whereas the relative dynamic pressure variation across the working section at the prism position is less than $0.3 \%$.

Investigations on two prisms with square cross-sections and sharp edges were performed, see Fig. 1a. Both prisms have side lengths of $D=60 \mathrm{~mm}$ and a span of $L=600 \mathrm{~mm}$, resulting in an aspect ratio of $\mathrm{AR}=L / D=10$. Each model consists of two stainless steel partially hollow half models, joined together with screws of which the heads were removed by milling, as shown in Fig. 1b. All surfaces were subsequently polished to reduce geometrical imperfections. This led to a non-dimensional equivalent sand-grain surface roughness, based on the algorithm by Adams et al. (2012), of $k_{s} l D \approx 4 \times 10^{-5} \pm 4.0 \times 10^{-6}$ for the smooth sharp-edged prism (labelled hereafter as "smooth prism"). The surfaces of the second prismatic model (labelled hereafter as "rough prism") were roughened using a plasmatic metal coating process, which resulted in a Gaussian-distributed relative surface roughness in both spanwise and circumferential directions of $k_{s} / D=1 \times 10^{-3} \pm 1.2 \times 10^{-4}$. The latter roughness value is typical for hard marine fouling on submerged surfaces of support columns of large floating offshore structures and wind turbines after a few years of operation. The prisms were mounted horizontally in the test section, thereby spanning its complete width. Labyrinth seals were used to

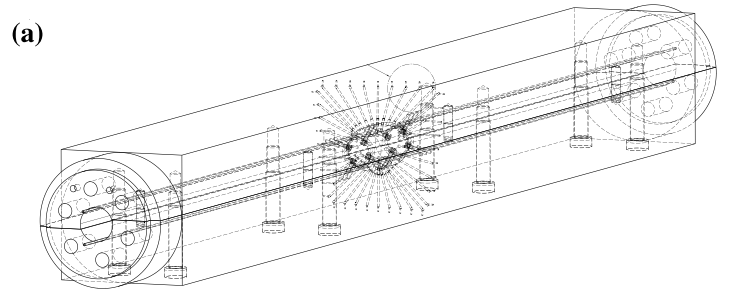

(b)
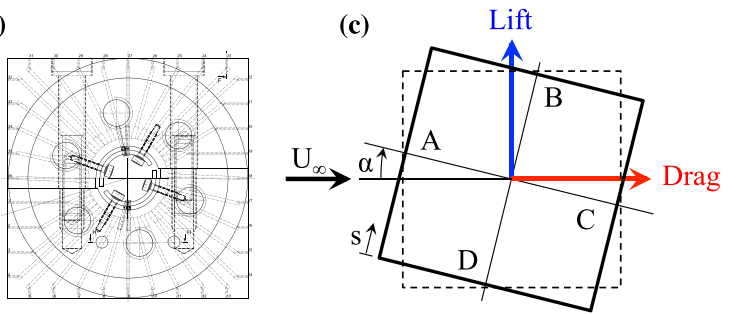

Fig. 1 Two-dimensional wind tunnel test model $(D=60 \mathrm{~mm}, L=$ $600 \mathrm{~mm}$ ) with the distribution of the static pressure taps over the midspan cross-section, as well as the numeration of the surfaces and the definition of the aerodynamic force vectors and incidence angle 
minimise the amount of flow leakage through the small ring gaps between the model and the side walls. Through surfaceoil visualisations, it has been shown in van Hinsberg et al. (2018) that the influence of the limited aspect ratio of 10 on the two-dimensionality of the flow over prisms, and thus on the measured overall aerodynamic loading, is relatively small as only at both most outer parts of the model a threedimensional flow was present, which was induced by the boundary layer on the side walls of the test section.

To obtain the surface pressure distribution at the midspan cross-section of each model, 36 static pressure taps (Fig. 1b) are connected through small stainless steel and plastic polyester tubes to a temperature-compensated electronic differential pressure measurement unit, having a range of $\pm 100 \mathrm{kPa}$ and a $0.15 \%$ uncertainty in the full scale. Their positions on the prism surface are given by the nondimensional circumferential coordinate $s / D$ with $s / D=0$ at the sharp edge between the surfaces $A$ and $D$ (Fig. 1c). Each pressure tap hole was shifted in the spanwise direction by a relative amount of $1.65 \mathrm{~mm}$ with respect to its direct neighbours to avoid an interference of the local pressure at a tap hole position with the wake generated by the pressure holes located upstream. The dynamic calibration of the pressure taps showed an upper cut-off frequency of about $860 \mathrm{~Hz}$. The mean base pressure coefficient $C_{p, b}$ for $\alpha=0^{\circ}$ was determined by taking the average of the pressure coefficient values obtained with the nine taps located on surface $C$ between s/D $=2.0$ and $\mathrm{s} / \mathrm{D}=3.0$, whereas all 18 taps on the surfaces $C$ and $D$, hence at $2.0 \leq \mathrm{s} / \mathrm{D} \leq 4.0$, were used to determine $C_{p, b}$ both at $\alpha=-22.5^{\circ}$ and at $\alpha=-45^{\circ}$. Both ends of each model were connected to rigid piezoelectric platform balances, located outside the test section, to obtain the time-dependent global lift and drag forces on the model with an uncertainty below $2 \%$. The resultant global mean lift and drag coefficients are defined as $C_{L}=\overline{L(t)} /\left(q_{\infty} D L\right)$ and $C_{D}=\overline{D(t)} /\left(q_{\infty} D L\right)$ with $\overline{L(t)}$ and $\overline{D(t)}$ the mean values of the global lift and drag forces and $q_{\infty}$ the dynamic pressure. These time-dependent signals were also used to extract additional information like the frequency spectra of the vortex shedding. With those power spectral densities (PSD), the Strouhal numbers could be determined, that are defined as $S t_{L}=f_{L} D / U_{\infty}$ and $S t_{D}=f_{D} D /\left(2 U_{\infty}\right)$, in which $f_{L}$ and $f_{D}$ are the main vortex shedding frequencies in the PSD based on the time series of the lift and drag force, respectively.

A pressure rake with 6 static pressure tubes and 52 pitot tubes was positioned at 6.25 diameters behind the model's spanwise centre axis to obtain useful data on the total nondimensional pressure loss in and the width of the near wake. The former is defined as the difference between the total pressure of the undisturbed flow $p_{0}$ and the total pressure at each location in the near wake $p$, divided by the dynamic pressure at inlet $q_{\infty}$, hence $\Delta p / q_{\infty}=\left(p_{0}-p\right) / q_{\infty}$. The latter is given by the distance between the two most outer pitot pressure tubes for which the value of the nondimensional pressure loss lies above the threshold of $5 \%$ of the maximum non-dimensional pressure loss, hence $\Delta p_{t h r}$ $=0.05 \Delta p_{\max }$. All data obtained with the pressure rake are assumed to have a $2 \%$ uncertainty.

Three angles of incidence were investigated, namely $0^{\circ},-22.5^{\circ}$ and $-45^{\circ}$. The resultant geometric wind tunnel blockage ratio lies between $0.10\left(\alpha=0^{\circ}\right)$ and $0.14(\alpha=$ $\left.-45^{\circ}\right)$. The measured velocities - and thus the Reynolds number, the Strouhal number and the global lift and drag coefficients-were thereupon corrected for wall interference effects by making use of the formulas of Allen and Vincenti (1944), whereas the blockage correction according to Roshko (1961) was applied to the static pressure coefficients and thus also to the 2D mean aerodynamic coefficients and the mean wake profiles, according to the following equations:

$$
\begin{gathered}
U_{\text {cor }}=U_{\text {uncor }}\left(1+0.25 C_{D, \text { uncor }} \frac{A}{Z_{w t}}\right. \\
\left.+0.85\left(\frac{A}{Z_{w t}}\right)^{2}\right) \\
C_{p, \text { cor }}=\left(C_{p, \text { uncor }}-1\right)\left(\frac{U_{\text {uncor }}}{U_{\text {cor }}}\right)^{2}+1
\end{gathered}
$$

with $A$ the area of the prism subject to the flow and $Z_{w t}$ the height of the test section. The resultant maximum applied corrections were in the order of $10 \%$. When placed in the test section, hence suspended in the piezoelectric balances, the first eigenfrequencies of both models were $f_{n, L}=340 \mathrm{~Hz}$ and $f_{n, D}=316 \mathrm{~Hz}$ in lift and drag directions, respectively. Since these values are well above the maximum occurring vortex-shedding frequencies, a lock-in of the vortex-shedding frequency with one of the eigenfrequencies could be avoided for most of the studied Reynolds numbers.

By varying both the free-stream velocity in the range of $\mathrm{U}_{\infty}=4-35 \mathrm{~m} / \mathrm{s}$ and the total air pressure inside the wind tunnel, $p_{0}$, between atmospheric and $10 \mathrm{MPa}$, a Reynoldsnumber range of $\operatorname{Re}_{D}=1 \times 10^{5}$ up to $1 \times 10^{7}$ could be covered. For each model configuration, a constant integration time of $T=30 \mathrm{~s}$ per measurement point was chosen for all recorded signals; the aerodynamic forces were thereby scanned with a sampling frequency of $f_{\text {scan }}=5 \mathrm{kHz}$. 


\section{Experimental results}

\subsection{Aerodynamics of smooth prisms: change in angle of incidence}

The mean aerodynamic force and pitch moment coefficients as a function of the Reynolds number are shown in Fig. 2a-c for the three investigated angles of incidence.

Figure $2 \mathrm{a}, \mathrm{b}$ presents both the global mean forces measured by the piezoelectric balances and those obtained by the circumferential integration of the mean pressure forces in the mid-span cross-section of the prism. The latter is equated according to

$$
\begin{gathered}
C_{l}=\frac{1}{D} \int_{s} C_{p} n_{Z} d s=\frac{1}{D} \sum_{i=1}^{36} C_{p, i} n_{Z, i} d s_{i} \\
C_{d}=\frac{1}{D} \int_{s} C_{p} n_{X} d s=\frac{1}{D} \sum_{i=1}^{36} C_{p, i} n_{X, i} d s_{i}
\end{gathered}
$$
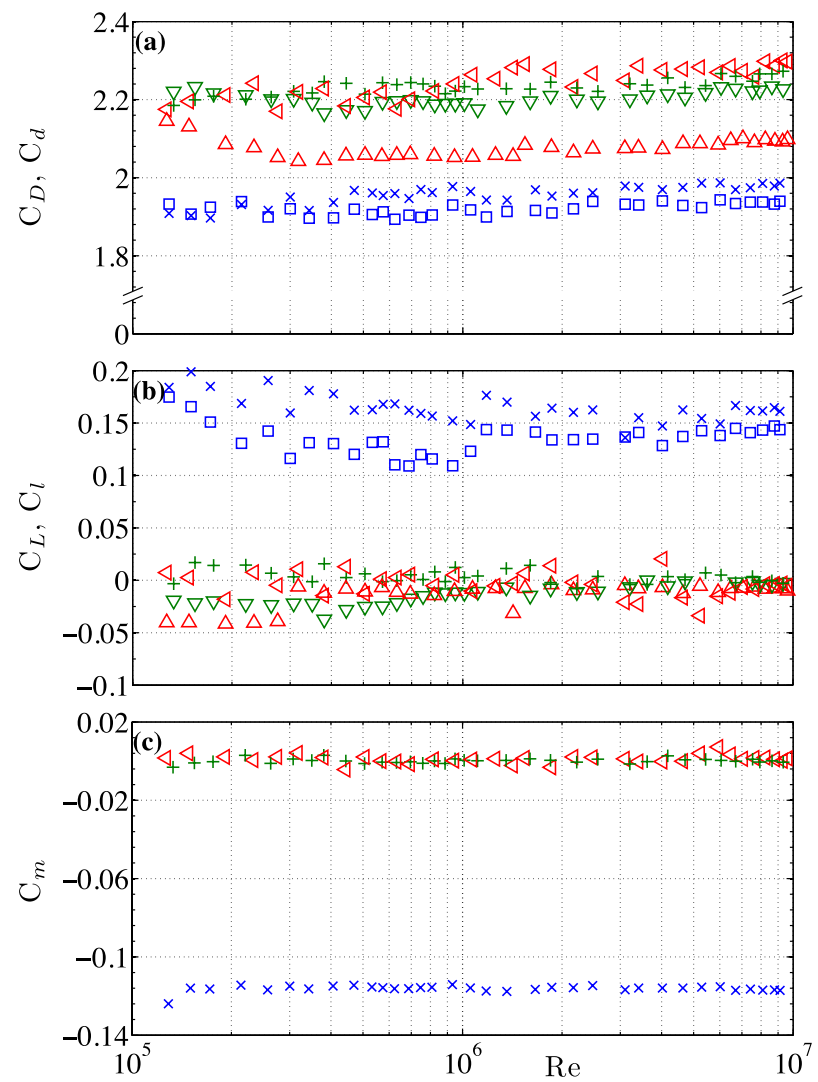

Fig. 2 D and global mean, as well as global fluctuating aerodynamic $\operatorname{drag}\left(C_{D}, C_{d}, \sqrt{\left(\overline{C_{D}^{\prime 2}}\right)}\right)$ and lift $\left(C_{L}, C_{l}, \sqrt{\left(\overline{C_{L}^{\prime 2}}\right)}\right)$ coefficients, 2D mean pitch moment $\left(C_{m}\right)$ coefficient and Strouhal number $\left(S t_{L}\right)$-based on
$C_{m}=\frac{1}{D^{2}} \int_{s} C_{p} n_{X} r d s=\frac{1}{D^{2}} \sum_{i=1}^{36} C_{p, i} n_{X, i} r_{i} d s_{i}$

with $D$ the side length of the prism, $d s_{i}=\left(s_{i+1}-s_{i-1}\right) / 2$ with $s_{i}$ the circumferential position of pressure tap $i$ measured along the peripheral of the prism according to Fig. 1c, $n$ the local normal vector on the surface of the prism at the position of pressure tap $i, X$ and $Z$ the downstream and upward-directed axes, respectively, with respect to the centre of the prism, $r_{i}$ the distance from the longitudinal centre axis up to the position of pressure tap $i$, and $C_{p, i}$ the pressure coefficient at the pressure tap $i$, defined as $C_{p, i}=\left(p_{i}-p_{\infty}\right) / q_{\infty}$ with $p_{i}$ the measured static pressure at pressure tap $i$ and $p_{\infty}$ the static pressure of the free-stream flow.

The overall similar values of the force coefficients for both measurement methods at each angle of incidence give rise to the presumption of the existence of a largely two-dimensional flow along the span of the prism, although a fine spanwise pressure distribution or PIV measurements are required to give detailed information on the amount of two-dimensionality along the span. It can be observed that all curves have a similar qualitative behaviour, characterised by a complete
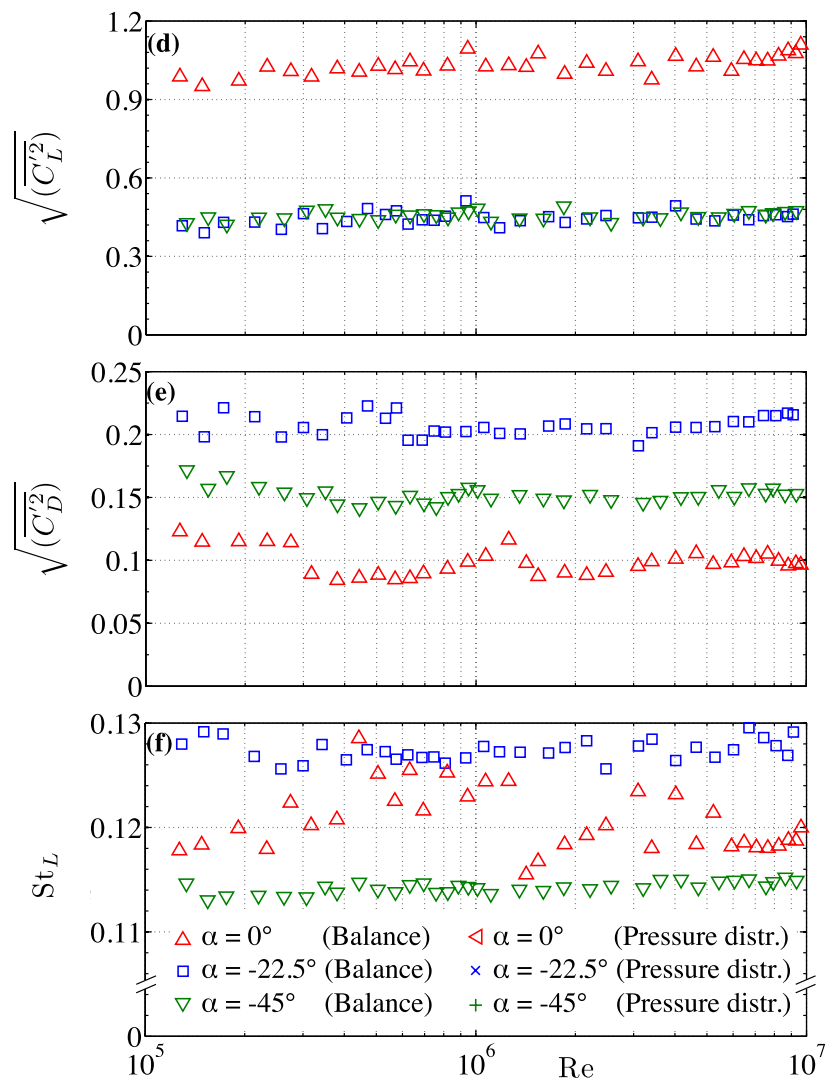

the main peak of the PSD of the time-dependent lift force in Fig. 5as function of the Reynolds number $R e_{D}$ for the two-dimensional smooth, sharp-edged square prism at $\alpha=0^{\circ},-22.5^{\circ}$ and $-45^{\circ}$ 
independency on the Reynolds number up to $10^{7}$. Regarding the $2 \mathrm{D}$ and global mean drag coefficients, only small changes in the values appear between the different investigated angles of incidence. As expected, the two- and three-dimensional mean lift forces equal zero for both "symmetric" configurations, i.e. $\alpha=0^{\circ}$ and $-45^{\circ}$, whereas steady constant mean lift coefficients in the range of $0.1-0.2$ are present for $\alpha=$ $-22.5^{\circ}$ over the complete Reynolds-number range. The same accounts for the two-dimensional mean pitch moment coefficient around the longitudinal axis of the prism in fig. 2c, which is based on eq. (2c): zero mean pitch moment coefficients for both "symmetric" angles of incidence and a stable negative mean pitch moment coefficient of $C_{m}=-0.116$ at all investigated Reynolds numbers for $\alpha=-22.5^{\circ}$.

As a result of the shedding of the free shear layers and the formation of the vortices in the near wake, air is being entrained into the base region of the prism. Per shedding period, two vortices with an opposite sign of circulation are formed, see, for example, Fig. 9 in van Oudheusden et al. (2005). The increase in both size and strength of each vortex during its formation induces a suction effect, as a result of which the opposing shear layer is drawn across the near wake (e.g. Lyn et al. (1995); Cao and Tamura (2016)). This process leads not only to a periodic interaction between the opposing vortices, but also to a periodic variation in rate of air entrainment into the base region. As a consequence, alternating out-of-plane pressure changes on both side surfaces of the prism are induced (Kurtulus et al. (2007)), which result then again in periodic fluctuating lift forces $C_{L}^{\prime}$ on the prism, their values being shown in Fig. 2d. The longer the time period during which each vortex is fed by circulation from its attached shear layer before being shed, the stronger and larger these vortices become, hence the larger the periodic changes in the surface pressure distribution will be. The values of $\sqrt{\left(\overline{C_{L}^{\prime 2}}\right)}$ can thus be seen as a measure of the intensity of the vortex shedding process. The results show that for $\alpha=0^{\circ}$ a constant level of high fluctuations in the lift coefficient in the order of $\sqrt{\left(\overline{C_{L}^{\prime 2}}\right)} \approx 1$ is present. Much lower lift fluctuations of about $\sqrt{\overline{\left(C_{L}^{\prime 2}\right)}}=0.45$ occur for both $\alpha=$ $-22.5^{\circ}$ and $-45^{\circ}$. The periodic vortex formation behind the prism induces not only pressure fluctuations in cross-flow direction, but also in the streamwise direction. Because the largest part of the total drag force on the prism is pressure drag, the component of the fluctuating surface pressure in the streamwise direction contributes to the fluctuating drag force. The values of $\sqrt{\left(\overline{C_{D}^{\prime 2}}\right)}$ belonging to the three investigated incidence angles in Fig. 2e lie in the range of 0.1 $\leq \sqrt{\left(\overline{C_{D}^{\prime 2}}\right)} \leq 0.22$, hence much lower than the lift fluctuations. Similar to the aerodynamic coefficients presented in the left column of Fig. 2, the fluctuations in both lift and drag force are highly Reynolds-number independent; a clear dependency on the angle of incidence is present though. The question, whether the Reynolds-number independence also counts for the fluctuations of the aerodynamic loads based on the fluctuating surface pressure coefficients, can unfortunately not be answered in this study, as the measured instantaneous pressure distributions on the prism surface were internally integrated in the electronic differential pressure measurement unit during the measurement; hence, only mean pressure values are available.

The power spectra (PSD) of the time-dependent lift and the drag forces for four Reynolds numbers per angle of incidence are presented in Fig. 3, together with values of the Strouhal numbers belonging to the main peak in each spectrum, hence the first harmonics (marked by the numbers 1 to 4 ), as well as the first subharmonics or the second or third harmonics (the numbers 5 to 8 ). The spectra of the lift fluctuations are characterised by one sharp narrow peak at the main vortex-shedding frequency. A lower and in particular for $\alpha=0^{\circ}$ broader (main) maximum is observed at $S t_{D}=2 S t_{L}$ in the power spectra of the drag fluctuations in the lower row of Fig. 3, which is for $\alpha=-22.5^{\circ}$ accompanied by a second peak at the first subharmonics of the main vortex-shedding frequency $f_{D}$, its Strouhal-number value corresponding to the $S t_{L}$-value in Fig. 3c. The Strouhal numbers based on the main frequency in the PSD of the time-dependent lift force signal are shown in Fig. $2 \mathrm{f}$ for the complete investigated Reynolds-number range. Whereas small changes in the values appear between the three angles of incidence, these three curves possess the same Reynoldsnumber independency as previously described for all other studied aerodynamic parameters.

\subsection{Aerodynamics of the rough prism case: change in angle of incidence}

Figure $4 \mathrm{a}-\mathrm{f}$ shows the variation of the $2 \mathrm{D}$ and global mean force coefficients, the 2D mean pitch moment coefficient, the fluctuations in lift and drag, and the Strouhal number with respect to the Reynolds number for the rough prism. Although the height of the surface roughness of this sharpedged prism is about a factor 25 larger than that of its smooth counterpart, a comparison with the results presented in Fig. 2 shows that the aerostatics of both prisms are remarkably similar. What probably catches the reader's eye right away is the complete absence of a correlation between the Reynolds number and the aerodynamic parameters. Just as was presented in the previous section for the smooth prism, all aerodynamic parameters for the rough prism remain, within the accuracy of the used measurement techniques, at constant values within the investigated Reynolds-number range as well.

The two- and three-dimensional drag coefficients for $\alpha=$ $0^{\circ}$ in Fig. $4 \mathrm{a}$ are about 2.2 and 2.0, respectively, hence slightly 

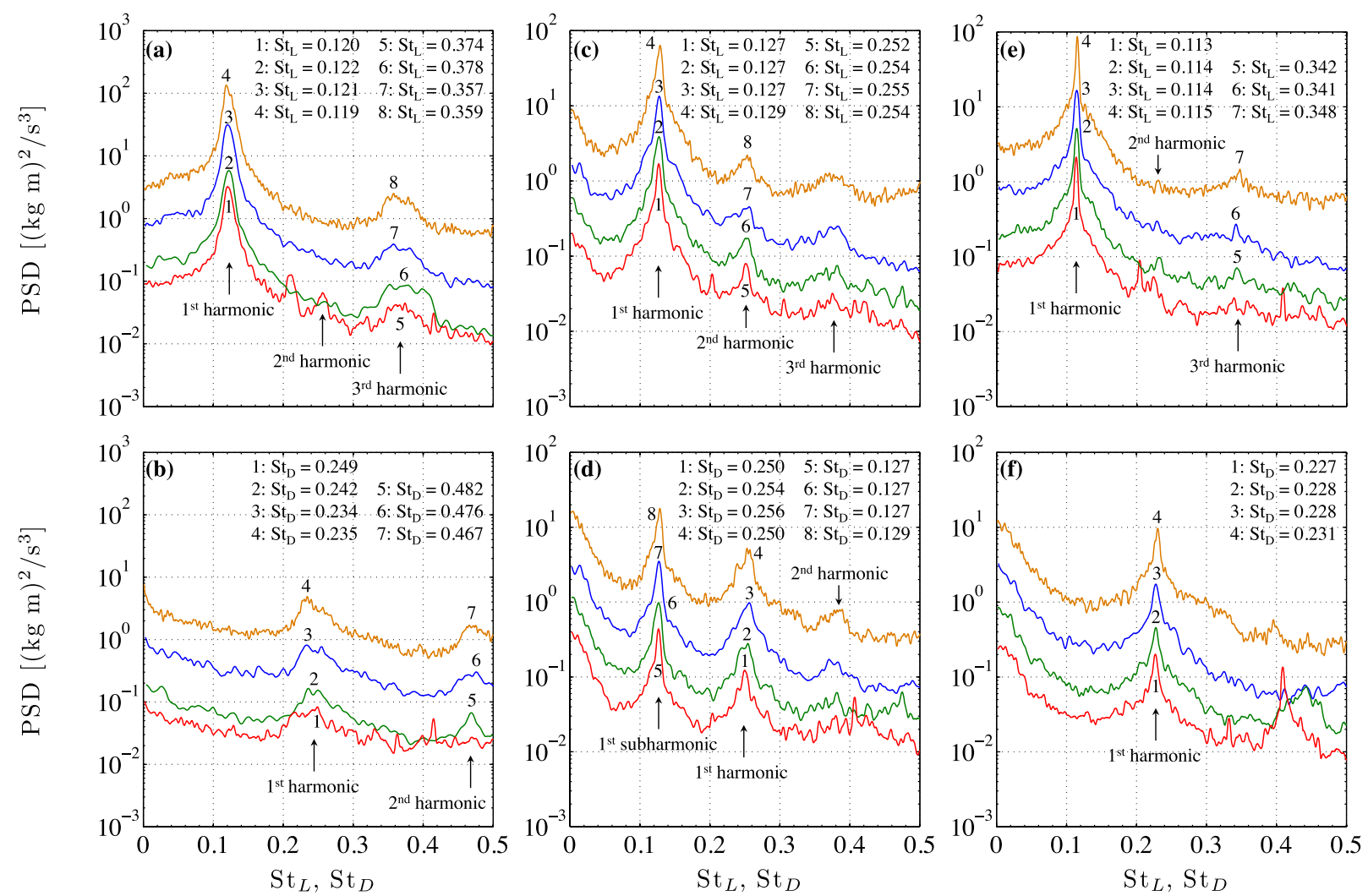

Fig. 3 Power spectral density of the time series of the lift (upper row) and drag forces (lower row) of the two-dimensional smooth, sharpedged square prism for selected Reynolds numbers at $\alpha=0^{\circ},-22.5^{\circ}$ and $-45^{\circ}$. The numbers $1-4$ mark the main peaks of each spectrum (first harmonics) and resemble the frequencies of the vortex shedding process in the base region behind the prism, whereas the numbers

lower than for the smooth prism. For $\alpha=-45^{\circ}$, no differences are found between both measurement methods though; both drag coefficients remain at a constant level of about $C_{D}=C_{d}=$ 2.2. Because of almost perfect symmetry in the surface pressure distributions between the upper and lower surfaces of the prism — as will be shown in section 4 - both $C_{l}$ and $C_{L}$ in Fig. $4 \mathrm{~b}$ and $C_{m}$ in Fig. $4 \mathrm{c}$ are close to zero for both angles of incidence.

Both the values of the fluctuations in lift $\left(\sqrt{\left(\overline{C_{L}^{\prime 2}}\right)} \approx 1.0\right.$ at $\alpha=0^{\circ}$ and $\sqrt{\left(\overline{C_{L}^{\prime 2}}\right)}=0.45$ for at $\left.\alpha=-45^{\circ}\right)$ and in drag $\left(\alpha=0^{\circ}: \sqrt{\left(\overline{C_{D}^{\prime 2}}\right)} \approx 0.1, \alpha=-45^{\circ}: \sqrt{\left(\overline{C_{D}^{\prime 2}}\right)} \approx 0.15\right)$, as well as the Strouhal numbers $\left(S t_{L} \approx 0.125\right.$ at $\alpha=0^{\circ}$ and $S t_{L}=$ 0.115 at $\alpha=-45^{\circ}$ ) in Fig. $4 d-$ f coincide perfectly well with the constant values obtained for the smooth prism in Fig. 2.

The PSD of the lift and drag forces for four selected Reynolds numbers are presented in Fig. 5. The narrow peak in the power spectra of the lift fluctuations at $S t_{L}=0.125$ and 0.115 for, respectively, $\alpha=0^{\circ}$ and $\alpha=-45^{\circ}$ and a broader and about on order of magnitude lower maximum in the power spectra of the drag fluctuations at twice the main vortex-shedding
5-8 mark the first subharmonics or the second or third harmonics. (a)-(b): $\alpha=0^{\circ},-\operatorname{Re}_{D}=2.96 \times 10^{5},-\operatorname{Re}_{D}=8.29 \times 10^{5},-\operatorname{Re}_{D}=3.10 \times$ $10^{6},-\operatorname{Re}_{D}=7.99 \times 10^{6} ;$ (c)-(d): $\alpha=-22.5^{\circ},-\operatorname{Re}_{D}=3.00 \times 10^{5},-\operatorname{Re}_{D}$ $=8.08 \times 10^{5},-\operatorname{Re}_{D}=3.10 \times 10^{6},-\operatorname{Re}_{D}=8.09 \times 10^{6} ;$ (e)-(f): $\alpha=-45^{\circ}$, $-\operatorname{Re}_{D}=3.08 \times 10^{5},-\operatorname{Re}_{D}=8.23 \times 10^{5},-\operatorname{Re}_{D}=3.21 \times 10^{6},-\operatorname{Re}_{D}=$ $7.93 \times 10^{6}$

frequency of $S t_{D}=2 S t_{L} \approx 0.255\left(\alpha=0^{\circ}\right)$ and $0.229(\alpha=$ $\left.-45^{\circ}\right)$ can be observed very clearly. A comparison with Fig. 3 shows that the current values of the Strouhal numbers at $\alpha=$ $0^{\circ}$ are slightly higher than for the smooth prism at practically equal Reynolds numbers, whereas at $-45^{\circ}$ angle of incidence a perfect equivalence of the values of both $S t_{L}$ and $S t_{D}$ between the two prisms is found.

\section{Discussion}

Based on the experimental data presented in Sect. 3, the differences in the static aerodynamics between the various angles of incidence of both prisms will be discussed in more detail hereafter. The focus lies hereby on the mean surface pressure distributions and the base pressure coefficient $C_{p, b}$, presented in Figs. 6 and 7, respectively, the mean wake profile (Figure 8), the aerodynamic coefficients and the Strouhal number.

The high $2 \mathrm{D}$ and global mean drag coefficients of $C_{d} \approx$ $C_{D}=2.0-2.2$ for both prisms at $\alpha=0^{\circ}$ in Figs. 2a and 4a are due to a combination of two phenomena: on the one hand 

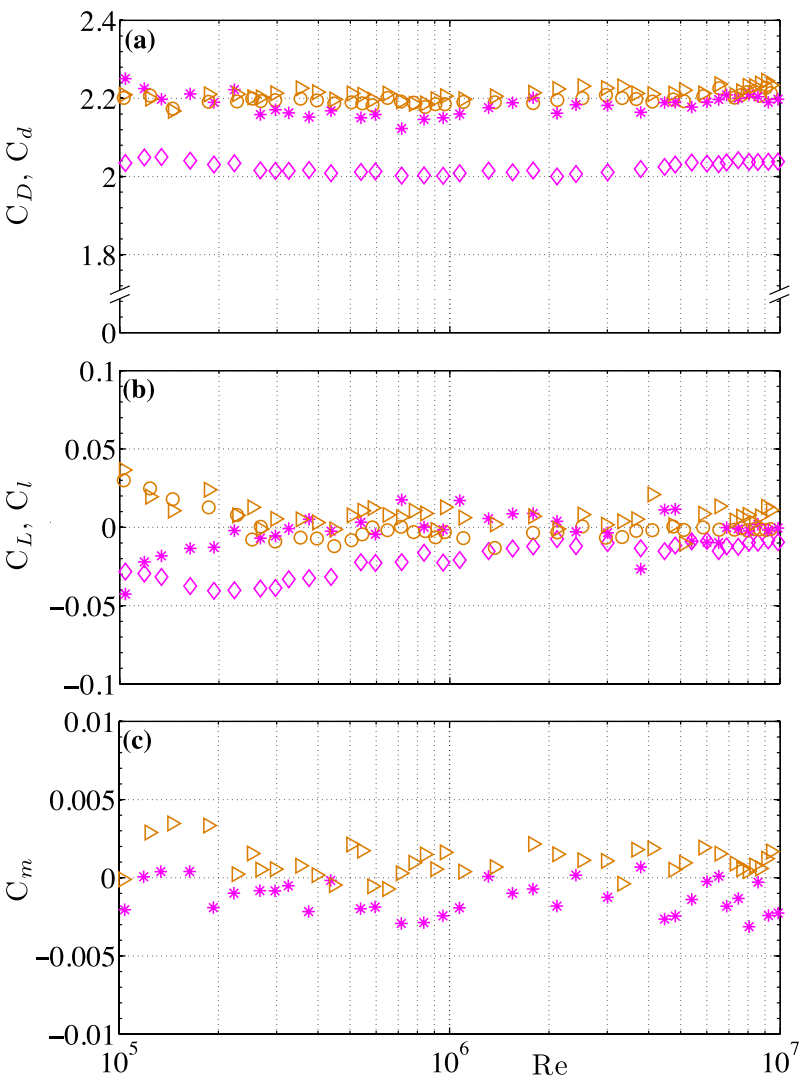

Fig. $42 \mathrm{D}$ and global mean, as well as global fluctuating aerodynamic $\operatorname{drag}\left(C_{D}, C_{d}, \sqrt{\left(\overline{C_{D}^{\prime 2}}\right)}\right)$ and lift $\left(C_{L}, C_{l}, \sqrt{\left(\overline{C_{L}^{\prime 2}}\right)}\right)$ coefficients, 2D mean pitch moment $\left(C_{m}\right)$ coefficient and Strouhal number $\left(S t_{L}\right)$ - based on

the relatively high negative base pressure coefficients of $C_{p, b}$ $\approx-1.55$ on surface $C$ with respect to the pressures on the windward surface $A$ in Figs. 6a, $g$ and 7, and on the other hand a distinct lateral spacing of the free shear layers (presented, among others, in Luo et al. (1994); van Oudheusden et al. (2005); Sohankar (2006); Sohankar et al. (2015); Cao and Tamura (2016); Bai and Alam (2018), and Cao et al. (2020) for various Reynolds numbers), which leads to an even larger effective aerodynamic blockage ratio than the geometric blockage ratio of 0.10 . A clear indication for the occurrence of the latter phenomenon in the current study is the large value of the wake width of $Z_{\text {wake }} / D=6$ still present at 5.75 diameters downstream of the model's base surface $C$, as shown in Fig. $8 \mathrm{a}, \mathrm{d}$ for the smooth and rough prism, respectively.

Since for bluff body flows the surface pressure drag accounts for the larger part of the total drag force on the bluff body, it is not surprising that the drag coefficients obtained by integration of $C_{p, c y l}(s / D)$ according to equation (2b) lie relatively close to the spanwise-integrated drag coefficients obtained by the piezoelectric balances, as presented in Figs. 2a and 4a. Small discrepancies between the values of both methods for both prisms are the result of the negligence
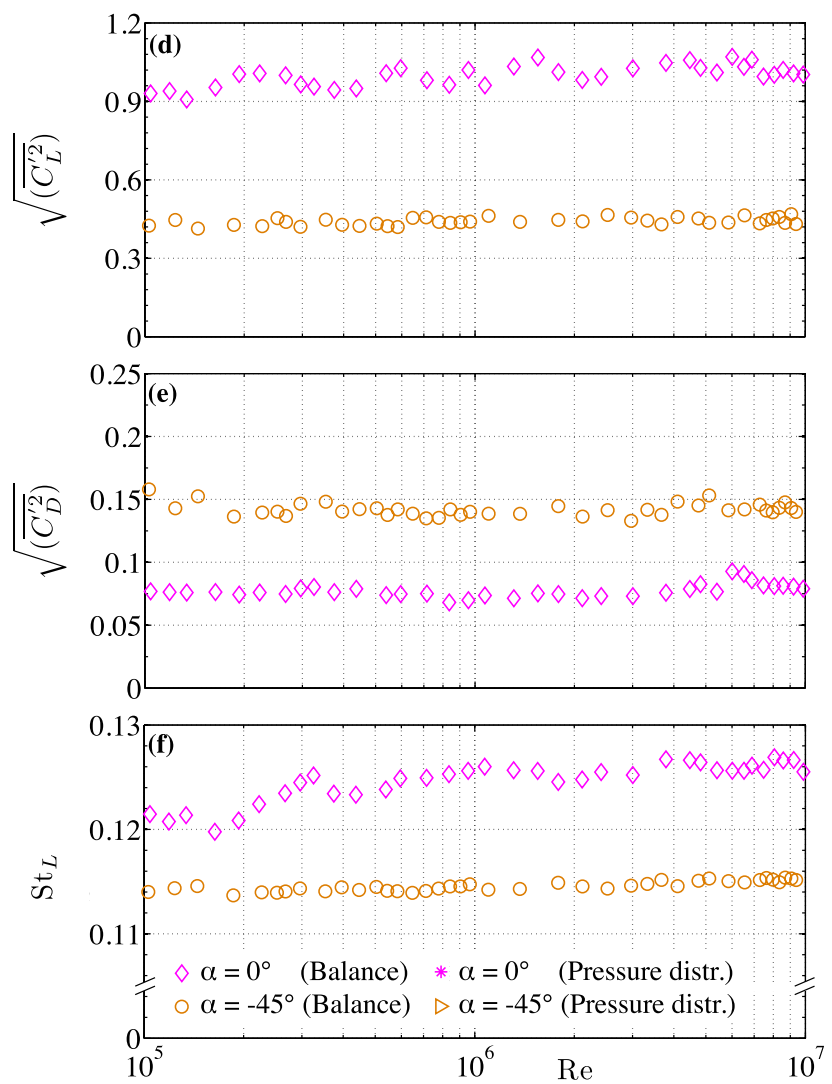

the main peak of the PSD of the time-dependent lift force in Fig. 5as function of the Reynolds number $R e_{D}$ for the two-dimensional rough, sharp-edged square prism at $\alpha=0^{\circ}$ and $-45^{\circ}$

of the component due to shear on the prism surfaces in Eq. (2b). Interesting to note is that for both prisms at $\alpha=45^{\circ}$ the 2D mean and the global mean drag coefficients obtain similar values, which implies equal shear components of the drag force on both prisms, hence being independent of the surface roughness height. For both prisms at $\alpha=0^{\circ}$, however, a $10 \%$ discrepancy in the drag coefficients between both measurement methods is obtained. This relatively large difference mainly results from the absence of pressure taps in the vicinity of the two upstream sharp edges at $s / D=$ 0 and 1 (Fig. 1b), owing to which the negative pressure peaks that are present at these edges could not be captured and were therefore not taken into account in equation (2b). Since the influence of these pressure peaks is included in the global drag forces, the resultant 2D mean drag coefficients therefore turn out to be somewhat larger than their global counterparts.

Durao et al. (1988); Lyn et al. (1995), and van Oudheusden et al. (2005) presented that the highest activity of the root mean square of the horizontal and vertical velocity fluctuations in the flow field around sharp-edged square prisms was found along the separated shear layers and at the wake 
Fig. 5 Power spectral density of the time series of the lift (upper row) and drag forces (lower row) of the two-dimensional rough, sharp-edged square prism for selected Reynolds numbers at $\alpha=0^{\circ}$ and $-45^{\circ}$. The numbers 1-4 mark the main peaks of each spectrum (first harmonics) and resemble the frequencies of the vortex shedding process in the base region behind the prism, whereas the numbers 5-8 mark the second or third harmonics. (a)-(b): $\alpha$ $=0^{\circ},-\operatorname{Re}_{D}=2.97 \times 10^{5},-\operatorname{Re}_{D}$ $=8.36 \times 10^{5},-\operatorname{Re}_{D}=3.01 \times 10^{6}$, - $\operatorname{Re}_{D}=8.06 \times 10^{6} ;$ (c)-(d): $\alpha=$ $-45^{\circ},-\operatorname{Re}_{D}=2.96 \times 10^{5},-\operatorname{Re}_{D}$ $=8.42 \times 10^{5},-\operatorname{Re}_{D}=2.97 \times 10^{6}$, $-\operatorname{Re}_{D}=7.98 \times 10^{6}$
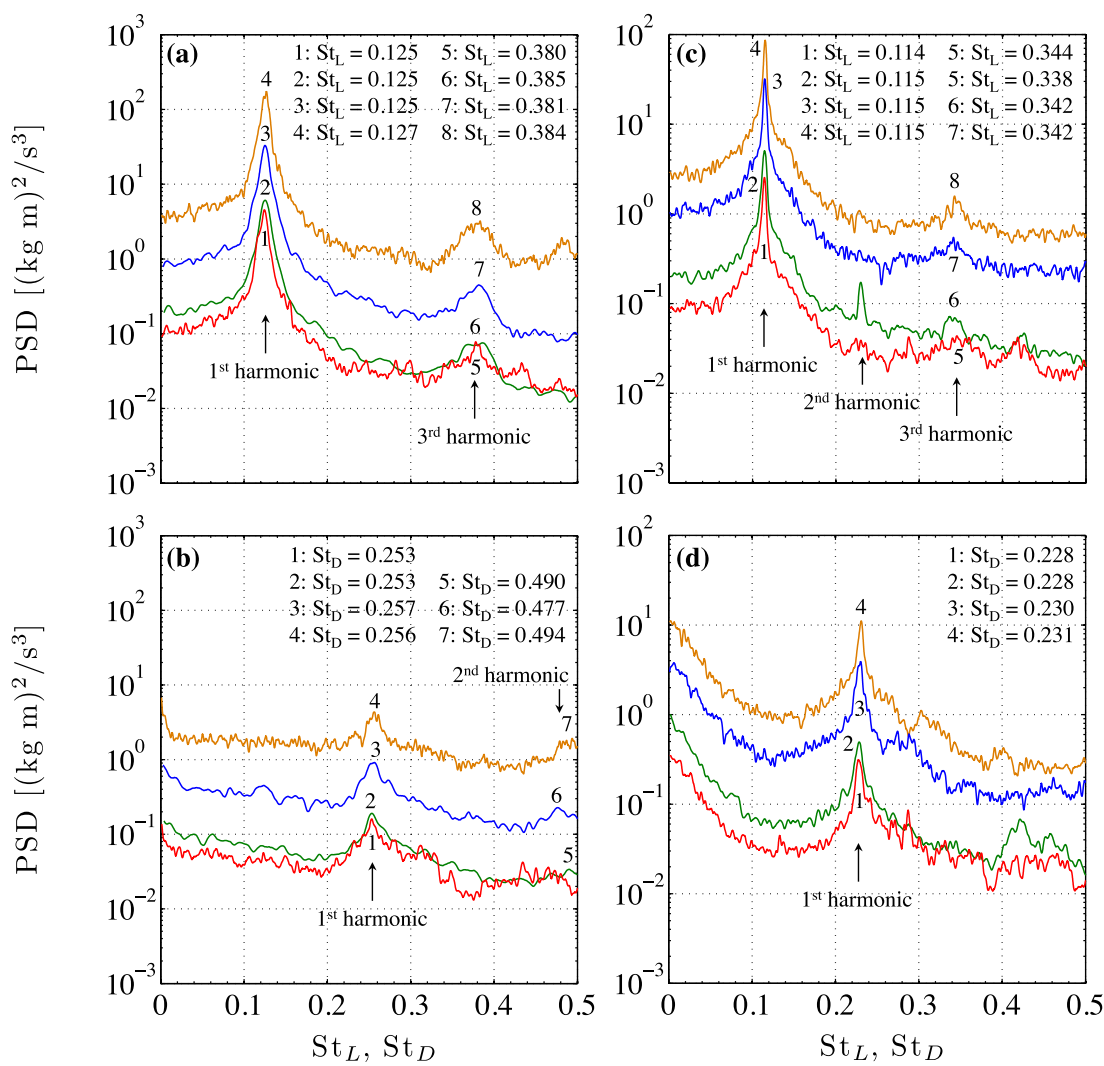

centreline just downstream of the four-way wake saddle point at which both wake division streamlines meet, respectively. As a consequence of the large-scale vortex shedding in the nearwake maximum velocity fluctuation levels as high as $60 \%$ for $u_{r m s} / U_{\infty}$ and $80 \%-90 \%$ for $v_{r m s} / U_{\infty}$ were measured at $0^{\circ}$ angle of incidence. The strong velocity fluctuations in crossflow direction in the base region enhance the entrainment of fluid into the wake during the vortex formation process and cause large periodic changes in the lift force on the prism, as is reflected by the high values of the fluctuations in the lift force in Fig. 2 d, i.e. $\sqrt{\left(\overline{C_{L}^{\prime 2}}\right)} \approx 1$. This high value agrees well with the results obtained by Carassale et al. (2014). Since the velocity fluctuations in the free-stream direction are about $25 \%$ smaller and their maximum values occur along the free shear layers, the periodic fluctuations in the drag force on the prism are much weaker, i.e. $\sqrt{\left(\overline{C_{D}^{\prime 2}}\right)} \leq 0.1$.

The cause of both a decrease of $C_{d}$ and $C_{D}$ as well as an increase of $C_{l}, C_{L}$ and $C_{m}$ at $\alpha=-22.5^{\circ}$ with respect to their values at $\alpha=0^{\circ}$ in Fig. 2 can be found in the change from a symmetric to an asymmetric flow around the prism by which an asymmetric pressure distribution on the surfaces of the prism is induced, as shown in Fig. 6c, d. Huang et al. (2010) and Sohankar et al. (2015) used the smoke-wire technique to visualise the changes in the flow structures around 2D sharpedged square prisms with incidence, whereby in the former study this was combined with the surface-oil flow technique to obtain the time-averaged flow patterns on all four prism surfaces. In both investigations, it was observed that at an incidence angle of about $\alpha=22^{\circ}$ a larger separation angle of the free shear layer at the leading edge of the downstreamdirected side surface (hence, surface $D$ in the current study) occurs than for $\alpha=0^{\circ}$. The combination of this increased "tilt-up angle" and the turning of this side face into the wake is responsible for an increase in the distance between the separated shear layer and its corresponding side surface. The negative pressure coefficients of $C_{p}<-1$ on the complete surface $D$ are an indication for the absence of a reattachment of the free shear layer to the side surface further downstream, in agreement with the flow patterns described by Huang et al. (2010) and Sohankar et al. (2015). Huang et al. (2010) furthermore noticed the appearance of a small trailing edge recirculation bubble on this surface (Figure 9 in Huang et al. (2010)). Although in the current study the possible presence of such a trailing edge separation bubble on surface $D$ cannot be confirmed by the measured surface pressure distribution only, those two aspects, i.e. increased "tilt-up angle" and trailing edge separation bubble-might be the cause for, respectively, the somewhat lower negative values of the surface pressure coefficients on surface $D$ between $\alpha$ $=0^{\circ}$ and $-22.5^{\circ}$ and the shift of the local minimum value of $C_{p}=-1.6$ at $s / D=3.4\left(\alpha=0^{\circ}\right)-s / D=3.1$ with $C_{p}=$ 

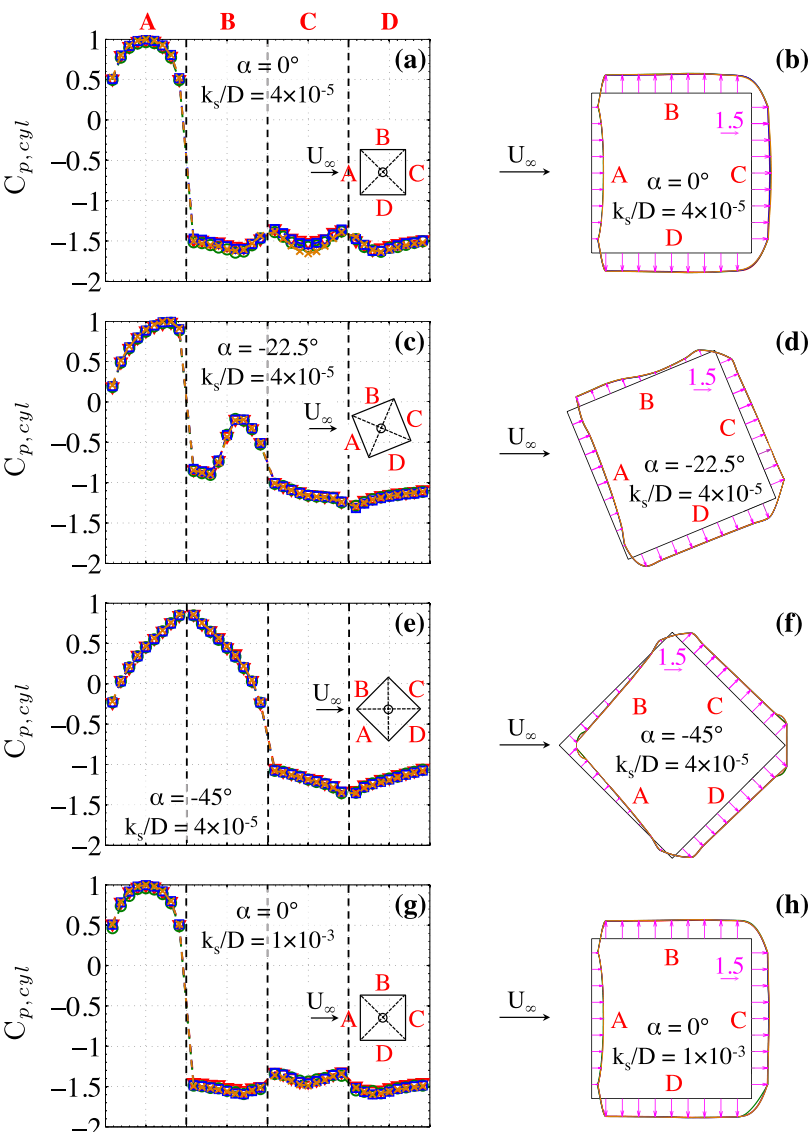

(h)
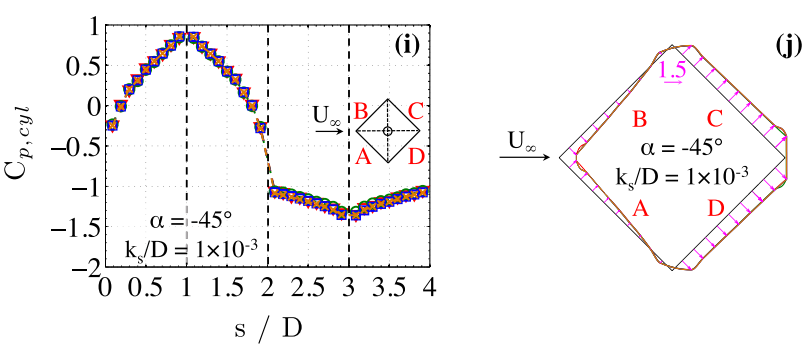

Fig. 6 Circumferential distribution of the mean pressure coefficients over the mid-span cross-section of the two-dimensional prism with a non-dimensional surface roughness of $k_{s} J D=4 \times 10^{-5}(a-f)$ and $k_{s} / D=1 \times 10^{-3}(g-j)$. The non-dimensional surface parameter $s / D$ increases in the clockwise direction with $s / D=0(=4)$ marking the edge between the surfaces $A$ and $D$. (a)-(b): $\alpha=0^{\circ}, \nabla \operatorname{Re}_{D}=2.96 \times$ $10^{5}$, 。 $\operatorname{Re}_{D}=8.29 \times 10^{5}, \square \operatorname{Re}_{D}=3.10 \times 10^{6}, \times \operatorname{Re}_{D}=7.99 \times 10^{6}$; (c)(d): $\alpha=-22.5^{\circ}, \nabla \operatorname{Re}_{D}=3.00 \times 10^{5}, \circ \operatorname{Re}_{D}=8.08 \times 10^{5}, \square \operatorname{Re}_{D}=3.10 \times$ $10^{6}, \times \operatorname{Re}_{D}=8.09 \times 10^{6} ;$ (e)-(f): $\alpha=-45^{\circ}, \nabla \operatorname{Re}_{D}=3.08 \times 10^{5}, \circ \operatorname{Re}_{D}$ $=8.23 \times 10^{5}, \square \operatorname{Re}_{D}=3.21 \times 10^{6}, \times \operatorname{Re}_{D}=7.93 \times 10^{6} ;(\mathrm{g})-(\mathrm{h}): \alpha=0^{\circ}$, $\nabla \operatorname{Re}_{D}=2.97 \times 10^{5}, \circ \operatorname{Re}_{D}=8.36 \times 10^{5}, \square \operatorname{Re}_{D}=3.01 \times 10^{6}, \times \operatorname{Re}_{D}=$ $8.06 \times 10^{6}$; (i)-(j): $\alpha=-45^{\circ}, \nabla \operatorname{Re}_{D}=2.96 \times 10^{5}, \circ \operatorname{Re}_{D}=8.42 \times 10^{5}, \square$ $\operatorname{Re}_{D}=2.97 \times 10^{6}, \times \operatorname{Re}_{D}=7.98 \times 10^{6}$

$-1.3\left(\alpha=-22.5^{\circ}\right)$, as shown in Fig. 6a, b. On the opposite side surface, the increased proximity of the shear layer upon separation at the edge between the surfaces $A$ and $B$ (hence, at $s / D=1.0$ in Fig. 1c) to its corresponding side surface $B$ leads to a reattachment to this surface further downstream

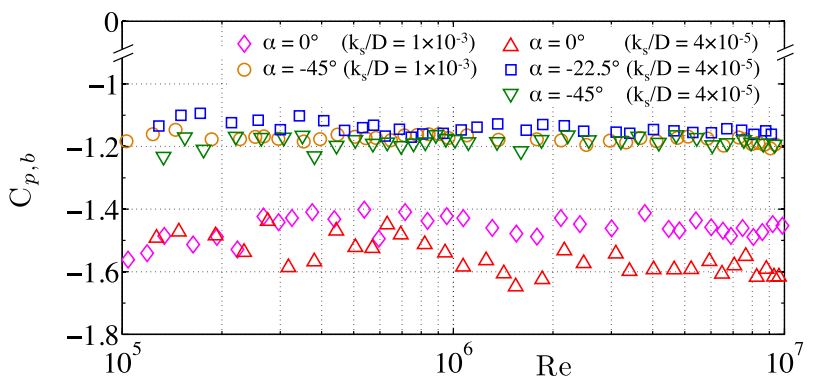

Fig. 7 Reynolds number dependency of the mean base pressure coefficient for the two-dimensional smooth and rough, sharp-edged square prisms

(Luo et al. (1994); Huang et al. (2010); Huang and Lin (2011); Sohankar et al. (2015)). Both Huang et al. (2010) and Sohankar et al. (2015) observed that the recirculation bubble that is formed on the upstream portion of this prism's side face at $\alpha \approx 22^{\circ}$ has a quasi-steady character: between shedding periods small changes in its size occur, and the separation bubble may sometimes even disappear completely over a short time period because of a temporary absence of the shear-layer reattachment to the side surface. In the study by Sohankar et al. (2015), a mean non-dimensional length of the recirculation bubble of $l_{\text {bubble }} / d=0.518$ was obtained at an incidence angle of $22.5^{\circ}$ for $R e_{D}=6776$. Although in the present study the surface pressure taps have a relatively large interspacing of $\Delta s / d=0.103$, it can be argued that the maximum pressure value of $C_{p}=-0.23$ at $s / D=1.6$ on surface $B$ in Fig. $6 \mathrm{~b}$ may coincide with the mean reattachment point of the free shear layer coming from the edge between the surfaces $A$ and $B$. Hence, this would result in a mean size of the recirculation bubble of $l_{\text {bubble }} / D \approx 0.6$ at $\alpha=-22.5^{\circ}$.

Although an increase of the geometric blockage ratio takes place when rotating the prism towards $\alpha=-22.5^{\circ}$, the reattachment of the lower free shear layer to the downstream portion of surface $B$ and its second separation at the surface trailing edge induce a straightening of the free shear layer in the base region of the prism (Figs. 2 and 3d in Huang et al. (2010) and Sohankar et al. (2015), respectively) that leads to a smaller size of the effective body in cross-flow direction seen by the incoming flow. This is confirmed by the reduction of the wake width behind the prism, being $Z_{\text {wake }} / D=$ 5.5 in comparison with $Z_{\text {wake }} / D=6$ for $\alpha=0^{\circ}$, as shown in Fig. 8b. In combination with a lower suction force in the base region (Figure 7), this leads to a lower drag coefficient of $C_{d}=C_{D}=1.9-2.0$. At the same time, about $45 \%$ higher periodic changes in the drag force on the prism are measured in comparison with the values at $\alpha=0^{\circ}$, as is shown in Fig. 2e. This increase is caused by the change in location of the free shear layer, which is formed at the trailing edge of surface $B$. Since this free shear layer is now located nearer 

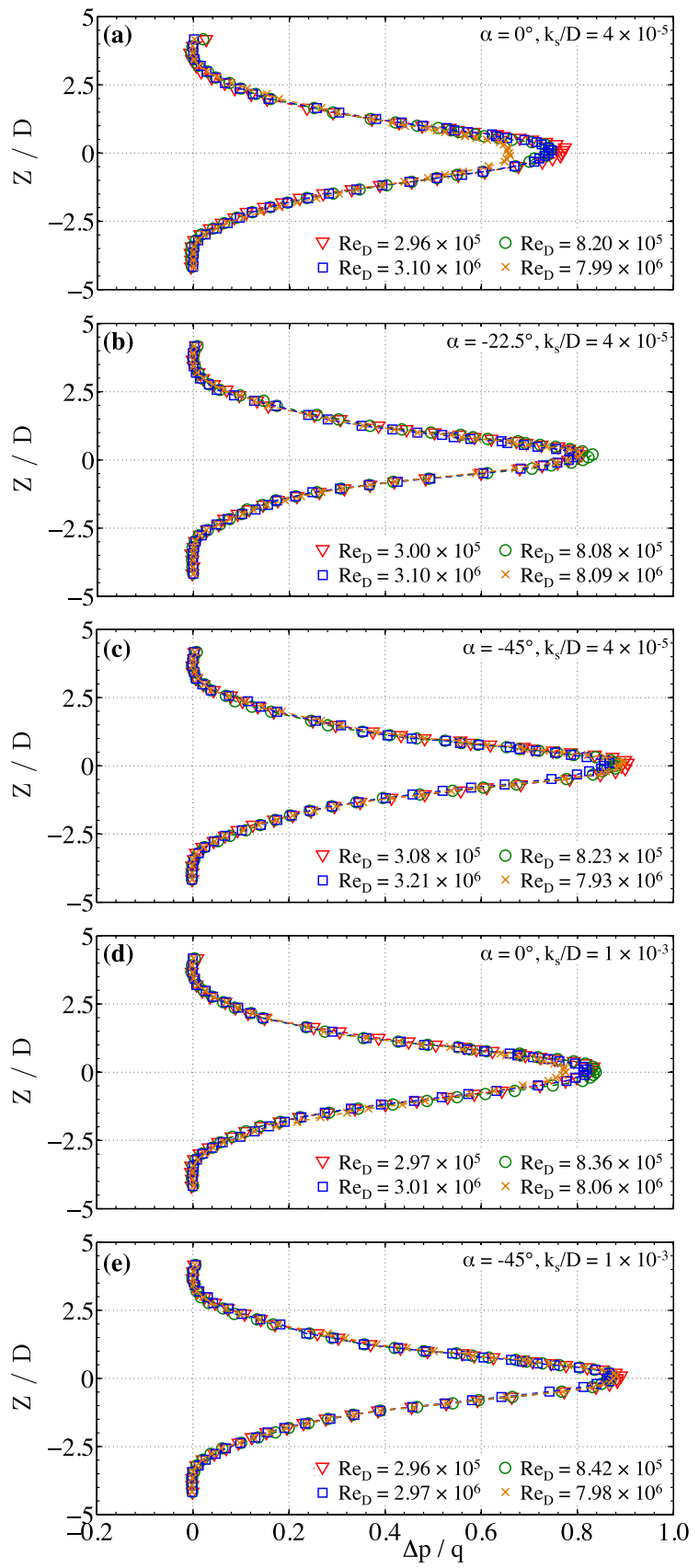

Fig. 8 Mean wake profiles of the two-dimensional prisms with a nondimensional surface roughness of $k_{s} / D=4 \times 10^{-5}(a-c)$ or $k_{s} / D=1 \times$ $10^{-3}(d-e) . X$ and $Z$ are, respectively, the downstream and upward directed axes seen from the centre of the prism, $\Delta p=\left(p_{0}-p\right)$ with $p_{0}$ the total pressure at the inlet, $p$ the pitot pressure at vertical locations in the near wake and $q$ the dynamic pressure at the inlet. The pressure rake is positioned at mid-span location 6.25 prism widths downstream of the prism's spanwise centre axis

to the wake centreline (Huang et al. (2010); Yen and Yang (2011); Sohankar et al. (2015)) and the largest fluctuations of the horizontal velocity component take place along both free shear layers, higher fluctuations of the surface pressure on the base of the prism in free-stream direction are now induced, which lead to higher r.m.s. values of the drag force. The appearance of a recirculation bubble above the upstream section of surface $B$ and its absence on surface $D$ furthermore induce a highly asymmetric flow pattern around the prism with strong differences between the shapes of the two large counter-rotating wake vortices. This results in a clear imbalance between the mean pressure coefficients in the cross-flow direction below and above the prism, which then again induces a steady positive mean lift force with fluctuations, which are cut by more than half, see Fig. $2 b$, d. The reason of the large jump in the values of $\sqrt{\left(\overline{C_{L}^{\prime 2}}\right)}$ from 1.0 for $\alpha=0^{\circ}$ to 0.45 for $\alpha=-22.5^{\circ}$ is most probably the turning of the surfaces $C$ and $D$ into the wake. These downstream-directed surfaces can be seen as an afterbody that is extruded downstream into the wake. It is assumed that the presence of this afterbody not only shifts the region with the highest vertical components of the velocity fluctuations on the wake centreline in downstream direction, but also causes a decrease in the amount of fluid that is entrained into the wake. The combined effect leads to a weaker intensity with which the vortices are shed from the prism and thus to smaller periodic changes in the lift force on the prism.

Among the three investigated angles of incidence, the highest mean drag coefficient of $C_{d}=C_{D} \approx 2.25$ is measured for the second "symmetric" angle of incidence of $\alpha=-45^{\circ}$. The combined effect of the largest possible geometric blockage ratio of 0.14 and the wide spreading in cross-flow direction of the free shear layers which separate from the edges at $s / D=0.0$ and 2.0 (Dutta et al. 2003; Ozgoren 2006; Huang et al. 2010; Yen and Yang 2011; Sohankar et al. 2015) induces a larger effective aerodynamic blockage ratio than at both other investigated angles of incidence, as well as larger vortices in the near wake (Huang et al. (2010); Yen and Yang (2011)). In Huang et al. (2010), it is for example shown that for $R e_{D} \leq 7.7 \times 10^{4}$ the wake width at a distance of about $0.5 D$ downstream of their sharp-edged prism is $1.25 D$ and $1.67 D$ for $\alpha=0^{\circ}$ and $45^{\circ}$, respectively. Using the smoke visualisation technique in their wind tunnel experiments, Sohankar et al. (2015) obtained similar values at $D / 4$ downstream of their sharp-edged prism for Reynolds numbers between $6.8 \times 10^{3}$ and $8.2 \times 10^{3}$. Because the suction force at the base of both prisms is somewhat weaker in comparison with $\alpha$ $=0^{\circ}$ (Figs. 6e, $\mathrm{i}$ and 7) and the wake widths have equal values at $\alpha=0^{\circ}$ and $-45^{\circ}$ (Fig. 8), it is assumed that the high values for the global and 2D drag coefficients are mainly related to the large effective aerodynamic blockage ratio.

The large lateral spacing of both free shear layers (Dutta et al. (2003); Huang et al. (2010); Yen and Yang (2011)) furthermore causes the values for $\sqrt{\left(\overline{C_{D}^{\prime 2}}\right)}$ to drop to a similar level as was found for $\alpha=0^{\circ}$. Interesting to note is furthermore that although the size of the afterbody present in the wake is largest for $\alpha=-45^{\circ}$, it has, according to the data 
presented in Figs. 2d and 4d, no influence at all on the values

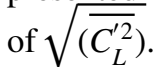

For comparison purposes, Table 1 lists for a wide range of Reynolds numbers in the shear layer transition II regime a collection of the aerodynamic force coefficients and the Strouhal number found in the literature on sharp-edged square prisms at the same angles of incidence as were selected for the current study. Despite differences in blockage ratio, aspect ratio, free-stream turbulence intensity and Reynolds number, the measured values for both the global mean and 2D mean lift and drag coefficients in the current experiment agree relatively well with most of the values obtained by others. Large differences only exist for $C_{d}$ measured by Norberg (1993) at $\alpha=22.5^{\circ}$, here obtained through a linear interpolation of his results for $20^{\circ}$ and $25^{\circ}$ angle of incidence, and at $\alpha=45^{\circ}$, for which differences as large as $\Delta C_{d}=-19 \%$ and $-29 \%$, respectively, are present, and those obtained by Huang and Lin (2011) at $\alpha=45^{\circ}$ with $\Delta C_{d}=$ $-17 \%$. Note furthermore the opposite sign of the currently measured lift coefficient at $\alpha=-22.5^{\circ}$ as a consequence of the counterclockwise rotation of the prism in this study.

The Strouhal numbers measured in this study are roughly $5-10 \%\left(\alpha=0^{\circ}\right), 10-35 \%\left(\alpha=-22.5^{\circ}\right)$ and $50-60 \%(\alpha=$ $-45^{\circ}$ ) lower than the values found in the literature, with the exception of those obtained by Carassale et al. (2014). In most of the listed experimental studies, either a hot-wire probe Norberg (1993); Huang and Lin (2011); Yen and Yang (2011), the LDV technique Lyn et al. (1995); Brun et al. (2008a) or a pressure tap at the mid-point of a side face Luo et al. (1994) was used to measure the periodic velocity or pressure fluctuations at a single point in the wake or on the side surface of the prism. Hence, those Strouhal numbers are correlated with the vortex shedding process at one certain spanwise position on the prism. In contrast, Carassale et al. (2014) used a piezoelectric force balance to extract the main vortex-shedding frequency by plotting the PSD function of the time-dependent spanwise-integrated lift force, in the same way as has been described in section 2. Because of the finite aspect ratios of the prisms in all listed studies, a spanwise correlation of the vortex shedding process only occurs within each of the cell-like structures that are present along the span of the prism. Humphreys (1960); van Hinsberg (2015), and Nikitas et al. (2012), for example, have proven the existence of such cell-like structures on smooth and rough circular cylinders perpendicular and inclined to the incoming flow, respectively. Small temporal and spatial fluctuations in the circumferential pressure distribution between neighbouring cell-like structures affect locally the vortex shedding process; hence, small variations in the vortex-shedding frequency can be found at different spanwise sections. By using piezoelectric balances to obtain the spanwise-integrated aerodynamic forces on the 2D prisms, an overall mean Strouhal number based on the mean of the vortex shedding frequencies over all cell-like structures is obtained, which differs from those measured with hot-wires, LDV or pressure fluctuations at a single point in the near wake behind the prism, hence correlated to one cell-like structure on the surface of the prism. Because the measurement method used by Carassale et al. (2014) corresponds to the one applied in the current study, it is not surprising that practically equal Strouhal numbers are found in both studies for all three angles of incidence.

\section{Closing remarks}

The presented experimental results are the first ones ever to show the aerostatic behaviour of 2D smooth and rough prisms with square cross-sections and sharp edges in low subsonic cross-flows for Reynolds numbers as high as $10^{7}$. Through wind tunnel experiments, the hypothesis of the existence of a Reynolds-number independency of the aerodynamics of this kind of bluff bodies, regardless of the value of the Reynolds number in the shear layer transition II regime, has been tested and proven to be correct. This counts both for smooth and for rough prism surfaces, as well as for angles of incidence in all three characteristic flow regimes, i.e. at $\alpha=0^{\circ}$ in the perfect separated flow regime, at $\alpha=-22.5^{\circ}$ in the separation bubble flow regime and at $\alpha=-45^{\circ}$ in the wedge flow regime. Independent of the surface roughness and Reynolds number, the primary boundary layer separation points remain fixed at both upstream sharp edges at incidence angles belonging to the first two flow regimes and at both shoulder edges at incidence angles in the wedge flow regime. Based on experimental proof, the question as to whether a Reynolds-number and/or surface-roughness dependency of their aerostatics exists can therefore be negated.

The challenging question, which still remains to be answered, is whether the insensitivity of the aerodynamics of smooth sharp-edged square prisms to variations in the Reynolds number can be assumed to exist for Reynolds numbers beyond $10^{7}$ as well. To artificially shift highReynolds-number flow phenomena towards physically low Reynolds numbers, which can still be achieved in wind and water tunnels, surface irregularities-like uniformly distributed dimples and grooves, solid particles, large sheets of glass or sand paper, or span-wise roughness stripes-have been applied in many experimental studies on the aerostatics of 2D circular cylinders in cross-flow, e.g. Güven et al. (1980); Achenbach and Heinecke (1981); Ribeiro (1991); Adachi (1997); Yamagishi and Oki (2004); Ma et al. (2019). In that way, an artificial transition and an earlier separation of the surface boundary layer could be provoked at lower Reynolds numbers. The main purpose of the high-pressure wind tunnel is actually to decouple 
Table 1 Mean force coefficients and Strouhal number for smooth prisms with square cross-sections and sharp edges obtained at different angles of incidence in the shear layer transition II regime for $R e_{D} \geq 10^{4}$

\begin{tabular}{|c|c|c|c|c|c|c|c|c|}
\hline Author & Blockage ratio & Aspect ratio & $\begin{array}{l}\text { Turbulence } \\
\text { intensity }\end{array}$ & $\alpha$ & $\operatorname{Re}_{D}$ & $C_{d}, C_{D}$ & $C_{l}, C_{L}$ & $S t$ \\
\hline \multirow[t]{3}{*}{ Present study } & $10 \%$ & 10 & $<0.8 \%$ & $0^{\circ}$ & $10^{5}-10^{7}$ & $\sim 2.15$ & 0 & 0.121 \\
\hline & & & & $-22.5^{\circ}$ & $10^{5}-10^{7}$ & 1.9 & $\sim 0.15$ & 0.127 \\
\hline & & & & $-45^{\circ}$ & $10^{5}-10^{7}$ & 2.25 & 0 & 0.114 \\
\hline \multirow[t]{3}{*}{ Naudascher and Fey (1981) } & $-a$ & 7.5 & $0.1 \%$ & $0^{\circ}$ & $1.1 \times 10^{5}$ & 1.99 & 0 & $-b$ \\
\hline & & & & $22^{\circ}$ & $1.1 \times 10^{5}$ & 1.85 & -0.21 & $-b$ \\
\hline & & & & $45^{\circ}$ & $1.1 \times 10^{5}$ & 2.2 & 0 & $-b$ \\
\hline \multirow[t]{4}{*}{ Norberg (1993) } & $<5 \%$ & $>50$ & $<0.06 \%$ & $0^{\circ}$ & $1.3 \times 10^{4}$ & 2.16 & 0 & 0.13 \\
\hline & & & & $20^{\circ}$ & $1.3 \times 10^{4}$ & 1.51 & -0.25 & 0.17 \\
\hline & & & & $25^{\circ}$ & $1.3 \times 10^{4}$ & 1.55 & -0.10 & 0.17 \\
\hline & & & & $45^{\circ}$ & $1.3 \times 10^{4}$ & 1.6 & 0 & 0.17 \\
\hline Luo et al. (1994) & $5 \%$ & 9.2 & $<0.5 \%$ & $0^{\circ}$ & $3.4 \times 10^{4}$ & 2.2 & 0 & 0.13 \\
\hline Lyn et al. (1995) & $7 \%$ & 9.75 & $2 \%$ & $0^{\circ}$ & $2.1 \times 10^{4}$ & 2.1 & $-b$ & 0.13 \\
\hline \multirow[t]{7}{*}{ Sohankar (2006) } & $6.25 \%$ & 4 & $0 \%$ & $0^{\circ}$ & $1.5 \times 10^{4}$ & $2.22^{c}$ & $-b$ & $0.124^{c}$ \\
\hline & & & & $0^{\circ}$ & $2.2 \times 10^{4}$ & $2.24^{c}, 2.19^{d}$ & $-b$ & $0.128^{c, d}$ \\
\hline & & & & $0^{\circ}$ & $5.0 \times 10^{4}$ & $2.20^{d}$ & $-b$ & $0.13^{d}$ \\
\hline & & & & $0^{\circ}$ & $1.0 \times 10^{5}$ & $2.20^{c}, 2.19^{d}$ & $-b$ & $0.128^{c, d}$ \\
\hline & & & & $0^{\circ}$ & $5.0 \times 10^{5}$ & $2.23^{c}$ & $-b$ & $0.128^{c}$ \\
\hline & & & & $0^{\circ}$ & $1.0 \times 10^{6}$ & $2.29^{c}$ & $-b$ & $0.128^{c}$ \\
\hline & & & & $0^{\circ}$ & $5.0 \times 10^{6}$ & $2.24^{c}$ & $-b$ & $0.124^{c}$ \\
\hline \multirow[t]{4}{*}{ van Oudheusden et al. (2007) } & $7.5 \%$ & 11.7 & $0.1 \%$ & $0^{\circ}$ & $2 \times 10^{4}$ & $2.18^{e}$ & $0.12^{e}$ & $-b$ \\
\hline & & & & $20^{\circ}$ & $2 \times 10^{4}$ & $1.80^{e}$ & $-0.32^{e}$ & $-b$ \\
\hline & & & & $25^{\circ}$ & $2 \times 10^{4}$ & $2.01^{e}$ & $-0.10^{e}$ & $-b$ \\
\hline & & & & $45^{\circ}$ & $2 \times 10^{4}$ & $2.32^{e}$ & $0.09^{e}$ & $-b$ \\
\hline Brun et al. (2008a) & $2 \%$ & 20 & $<0.8 \%$ & $0^{\circ}$ & $2 \times 10^{4}-3 \times 10^{5}$ & $2.1^{f}$ & $-b$ & $0.130-0.140$ \\
\hline \multirow[t]{8}{*}{ Huang and Lin (2011) } & $10 \%$ & 10 & $<0.2 \%$ & $0^{\circ}$ & $7.7 \times 10^{4}$ & 2.13 & 0 & $0.13^{g}$ \\
\hline & & & & $0^{\circ}$ & $9.4 \times 10^{4}$ & 2.09 & -0.03 & $0.13^{g}$ \\
\hline & & & & $20^{\circ}$ & $7.7 \times 10^{4}$ & 1.70 & -0.41 & $0.193^{g}$ \\
\hline & & & & $20^{\circ}$ & $9.4 \times 10^{4}$ & 1.63 & -0.46 & $0.19^{g}$ \\
\hline & & & & $25^{\circ}$ & $7.7 \times 10^{4}$ & 1.83 & -0.06 & $-b$ \\
\hline & & & & $25^{\circ}$ & $9.4 \times 10^{4}$ & 1.77 & -0.11 & $-b$ \\
\hline & & & & $45^{\circ}$ & $7.7 \times 10^{4}$ & 1.90 & 0 & $0.186^{g}$ \\
\hline & & & & $45^{\circ}$ & $9.4 \times 10^{4}$ & 1.83 & 0 & $0.183^{g}$ \\
\hline \multirow[t]{4}{*}{ Yen and Yang (2011) } & $4 \%$ & 4 & $0.3 \%$ & $0^{\circ}$ & $2.4 \times 10^{4}$ & 1.96 & -0.01 & 0.134 \\
\hline & & & & $0^{\circ}$ & $3.6 \times 10^{4}$ & 2.02 & -0.03 & 0.14 \\
\hline & & & & $45^{\circ}$ & $2.4 \times 10^{4}$ & 2.1 & 0 & 0.177 \\
\hline & & & & $45^{\circ}$ & $3.6 \times 10^{4}$ & 2.21 & 0 & 0.175 \\
\hline \multirow[t]{4}{*}{ Carassale et al. (2014) } & $-a$ & $9-40$ & $0.2 \%$ & $0^{\circ}$ & $3.7 \times 10^{4}$ & 2.07 & -0.01 & 0.124 \\
\hline & & & & $20^{\circ}$ & $3.7 \times 10^{4}$ & 1.92 & -0.29 & 0.133 \\
\hline & & & & $25^{\circ}$ & $3.7 \times 10^{4}$ & 2.07 & -0.06 & 0.125 \\
\hline & & & & $45^{\circ}$ & $3.7 \times 10^{4}$ & 2.34 & -0.01 & 0.115 \\
\hline
\end{tabular}

${ }^{a}$ no specifications mentioned by the authors

${ }^{b}$ no data available

${ }^{c}$ 3D large eddy simulation with the dynamic one-equation subgrid scale model

${ }^{d}$ 3D large eddy simulation with the Smagorinsky subgrid scale model

${ }^{e}$ obtained from PIV velocity field data through application of a control-volume approach

${ }^{f}$ obtained with 2D HWA measurements for $2.2 \times 10^{4} \leq \operatorname{Re}_{D} \leq 1 \times 10^{5}$, see Goossens et al. (2004)

${ }^{g}$ from Huang et al. (2010) 
the effects of both influencing parameters, i.e. Reynolds number and surface roughness height, by varying the total air pressure, while keeping all other boundary conditions like cylinder diameter, surface texture and height, object dimensions and free-stream turbulence intensity constant. The data of two previous experimental investigations, conducted in this wind tunnel, on the flow around 2D smooth and rough circular cylinders (van Hinsberg (2015)) and square-section prisms with non-dimensional edge radii of $r / D=0.29$ and 0.16 (van Hinsberg et al. (2018)) can nevertheless be used to make a statement on the presumed aerostatic behaviour of smooth sharp-edged square prisms at ultra-high Reynolds numbers beyond $R e_{D}=10^{7}$. Both previous experiments have shown that an increase in surface roughness height from $k_{s} / D=10^{-5}$ (smooth) to $k_{s}$ $/ D=10^{-3}$ (rough) results in a shift of the boundaries of all flow regimes to lower Reynolds numbers by a factor of $\operatorname{Re}_{D, \text { smooth }} / \operatorname{Re}_{D, \text { rough }}=1.8$ for circular cylinders $(r / D=$ 0.5 ) and by a factor of $\operatorname{Re}_{D, \text { smooth }} / \operatorname{Re}_{D, \text { rough }}=2.9$ and 4.1 for square-section prisms with edge radii of $r / D=0.29$ and 0.16 , respectively. By extrapolating the exponential fit through the points $\left(r / D, \operatorname{Re}_{D \text {,smooth }} / \operatorname{Re}_{D, \text { rough }}\right)$, a theoretical factor of $\operatorname{Re}_{D, \text { smooth }} / \operatorname{Re}_{D, \text { rough }} \approx 6$ would be achieved for the current sharp-edged square prism with $r / D=0$. By mapping the aerostatic results of the rough sharp-edged square prism onto the measured data of its smooth counterpart, it is to be expected that up to an ultra-high Reynolds number of about $\operatorname{Re}_{D}=60$ million no significant variation with Reynolds number will occur. Unfortunately, the author is not aware of any currently existing low-subsonic wind tunnel in which these kinds of Reynolds numbers can be reached to test this new hypothesis.

Acknowledgements Technical assistance from Markus Löhr of DNW (German Dutch Wind Tunnels) is gratefully acknowledged. Systemhaus Technik and Deharde are recognised for the construction and manufacturing of the wind tunnel models, respectively. Finally, the author would like to thank the reviewers for their fruitful comments and suggestions.

Funding Open Access funding enabled and organized by Projekt DEAL.

\section{Compliance with ethical standards}

Conflicts of interest The author declares that he has no conflict of interest.

Open Access This article is licensed under a Creative Commons Attribution 4.0 International License, which permits use, sharing, adaptation, distribution and reproduction in any medium or format, as long as you give appropriate credit to the original author(s) and the source, provide a link to the Creative Commons licence, and indicate if changes were made. The images or other third party material in this article are included in the article's Creative Commons licence, unless indicated otherwise in a credit line to the material. If material is not included in the article's Creative Commons licence and your intended use is not permitted by statutory regulation or exceeds the permitted use, you will need to obtain permission directly from the copyright holder. To view a copy of this licence, visit http://creativecommons.org/licenses/by/4.0/.

\section{References}

Achenbach E (1968) Distribution of local pressure and skin friction around a circular cylinder in cross-flow up to $\mathrm{Re}=5 \times 10^{6}$. J Fluid Mech 34:625-639

Achenbach E, Heinecke E (1981) On vortex shedding from smooth and rough cylinders in the range of Reynolds numbers $6 \times 10^{3}$ to $5 \times 10^{6}$. J Fluid Mech 109:239-251

Adachi T (1997) Effects of surface roughness on the universal Strouhal number over the wide Reynolds number range. J Wind Eng Ind Aerodyn 69-71:399-412

Adams T, Grant C, Watson H (2012) A simple algorithm to relate measured surface roughness to equivalent sand-grain roughness. Int J Mech Eng Mech 1:66-71

Allen HJ, Vincenti WG (1944) Wall interference in a two-dimensional flow wind tunnel with consideration of the effect of compressibility. Tech Rep 782, Nat Adv Comm Aero Report $155-184$

Amandolèse X, Hémon P (2010) Vortex-induced vibration of a square cylinder in wind tunnel. Comptes Rendus Mécanique $1: 12-17$

Bagbanci H, Kamakar D, Soares CG (2012) Review of offshore floating wind turbine concepts. In: Soares CG, Garbatov Y, Sutulo S, Santos T (eds) Maritime Engineering and Technology,. Taylor \& Francis Group, London UK

Bahrami A, Hacişevki H (2019) Comparison of flow structures in the wake region of a square cylinder or a $U$ shape cylinder. Ocean Eng 187:106211-1-11

Bai H, Alam MM (2018) Dependence of square cylinder wake on Reynolds number. Phys Fluids 30:015102-1-19

Bearman PW (1969) On vortex shedding from a circular cylinder in the critical Reynolds number regime. J Fluid Mech 37:577-585

Brun C, Aubrun S, Goossens T, Ravier P (2008a) Coherent structures and their frequency signature in the separated shear layer on the sides of a square cylinder. Flow Turbul Combust 81:97-114

Caliskan S (2014) Experimental investigation of heat transfer in a channel with new winglet-type vortex generators. Int J Heat Mass Transf 78:604-614

Cao Y, Tamura T (2016) Large-eddy simulations of flow past a square cylinder using structured and unstructured grids. Comput Fluids 137:36-54

Cao Y, Tamura T, Kawai H (2020) Spanwise resolution requirements for the simulation of high-Reynolds-number flows past a square cylinder. Comput Fluids 196(104320):1-16

Chen JM, Liu CH (1999) Vortex shedding and surface pressures on a square cylinder at incidence to a uniform air stream. Int J Heat Fluid Flow 20:592-597

Chen ZS, Liu SM, Yu XF, Ma CM, Liu L (2017) Experimental investigations on VIV of bridge deck sections: a case study. J Civ Eng 21(7):2821-2827

Cheng Z, Madsen HA, Chai W, Gao Z, Moan T (2017) A comparison of extreme structural responses and fatigue damage of semi-submersible type floating horizontal and vertical axis wind turbines. Renew Energy 108:207-219

Demartino C, Koss HH, Georgakis CT, Ricciardelli F (2015) Effects of ice accretion on the aerodynamics of bridge cables. J Wind Eng Ind Aerodyn 138:98-119 
Desai A, Mittal S, Mittal S (2020) Experimental investigation of vortex shedding past a circular cylinder in the high subcritical regime. Phys Fluids 32:014105-1-18

Durao D, Heitor M, Pereira J (1988) Measurements of turbulent and periodic flows around a square cross-section cylinder. Exp Fluids 6:298-304

Dutta S, Muralidhar K, Panigrahi PK (2003) Influence of the orientation of a square cylinder on the wake properties. Exp Fluids $34: 16-23$

ESDU (1971) Fluid Forces, Pressures and Moments on Rectangular Blocks. Engineering Scoieties Data Unit, Data Item 71016

Farell C, Blessmann J (1983) On critical flow around smooth circular cylinders. J Fluid Mech 136:375-391

Forrest JS, Kaaria CH, Owen I (2016) Evaluating ship superstructure aerodynamics for maritime helicopter operations through CFD and flight simulation. The Aeronaut J 120(1232):1578-1603

Gatto A, Graham WR (2017) Investigation of unsteady surface pressures over a two-wheeled leading-gear model. J Aircr 54:272-283

Gonçalves RT, Rosetti GF, Fujarra ALC, Oliveira AC (2012) Experimental study on vortex-induced motions of a semi-submersible platform with four square columns, Part I: effects of current incidence angle and hull appendages. Ocean Eng 54:150-169

Gonçalves RT, Rosetti GF, Fujarra ALC, Oliveira AC (2013) Experimental study on vortex-induced motions of a semi-submersible platform with four square columns, Part II: effects of surface waves, external damping and draft condition. Ocean Eng 62:10-24

Goossens T, Brun C, Doris L, Hureau J (2004) Kelvin-Helmholtz structures in the separated shear layer on the top of a square cylinder: experimental and numerical investigation. 10th EUROMECH European turbulence conference, Trondheim, Norway

Güven O, Farell C, Patel VC (1980) Surface-roughness effects on the mean flow past circular cylinders. J Fluid Mech 98:673-701

Hacişevki H, Teimourian A (2015) Comparison of flow structures in the wake region of two similar normal flat plates in tandem and a square cylinder. Exp Therm Fluid Sci 69:169-177

Hall M, Buckham B, Crawford C (2014) Hydrodynamics-based floating wind turbine support platform optimization: a basis function approach. Renew Energy 66:559-569

Helgedagsrud TA, Bazilevs Y, Mathisen KM, Øiseth OA (2019) Computational and experimental investigation of free vibration and flutter of bridge decks. Computational Mech 63:121-136

van Hinsberg NP (2015) The Reynolds number dependency of the steady and unsteady loading on a slightly rough circular cylinder: from subcritical up to high transcritical flow state. J Fluids Struct 55:526-539

van Hinsberg NP, Schewe G, Jacobs M (2017) Experiments on the aerodynamic behaviour of square cylinders with rounded corners at Reynolds numbers up to 12 million. J Fluids Struct 74:214-233

van Hinsberg NP, Schewe G, Jacobs M (2018) Experimental investigation on the combined effects of surface roughness and corner radius for square cylinders at high Reynolds numbers up to $10^{7}$. J Wind Eng Ind Aerodyn 173:14-27

Hu JC, Zhou Y, Dalton C (2006) Effects of corner radius on the near wake of a square prism. Exp Fluids 40:106-118

Huang RF, Lin BH (2011) Effects of flow patterns on aerodynamic forces of a square cylinder at incidence. J Mech 27(03):347-355

Huang RF, Lin BH, Yen SC (2010) Time-averaged topological flow patterns and their influence on vortex shedding of a square cylinder in crossflow at incidence. J Fluids Struct 26:406-429

Humphreys JS (1960) On a circular cylinder in a steady wind at transition Reynolds numbers. J Fluid Mech 9(4):603-612

Igarashi T (1984) Characteristics of the flow around a square prism. Bull JSME 27(231):1858-1865

Irwin P, Denoon R, Scott D (2013) Wind tunnel testing of high-rise buildings: an output of the CTBUH wind engineering working group. Tech Rep 3038, Council on tall buildings and urbin habitat tech report

Ke J (2019) RANS and hybrid LES/RANS simulations of flow over a square cylinder. Adv Aerodyn 1:1-24

Knisely CW (1990) Strouhal numbers of rectangular cylinders at incidence, A review and new data. J Fluids Struct 4:371-393

Kurtulus DF, Scarano F, David L (2007) Unsteady aerodynamic forces estimation on a square cylinder by TR-PIV. Exp Fluids 42:185-196

Lee BE (1975) The effect of turbulence on the surface pressure field of a square prism. J Fluid Mech 69:321-352

Luo SC, Yazdani MG, Chew YT, Lee TS (1994) Effects of incidence and afterbody shape on flow past bluff cylinders. J Wind Eng Ind Aerodyn 53:375-399

Lyn DA, Einav S, Rodi W, Park JH (1995) A Laser-Doppler velocimetry study of ensemble-averaged characteristics of the turbulent near wake of a square cylinder. J Fluid Mech 304:285-319

Ma W, Liu Q, Macdonald JHG, Yan X, Zheng Y (2019) The effect of surface roughness on aerodynamic forces and vibrations for a circular cylinder in the critical Reynolds number range. J Wind Eng Ind Aerodyn 187:61-72

Mangrulkar CK, Dhoble AS, Pant PK, Kumar N, Gupta A, Chamoli S (2019) Thermal performance escalation of cross flow heat exchanger using in-line elliptical tubes. Exp Heat Transf. 1-26

Minguez M, Brun C, Pasquetti R, Serre E (2011) Experimental and high-order LES analysis of the flow in near-wall region of a square cylinder. Int J Heat Fluid Flow 32:558-566

Naudascher E, Fey JWB (1981) Exploratory study in damping of galloping vibrations. J Wind Eng Ind Aerodyn 8:211-222

Nikitas N, Macdonald JHG (2015) Aerodynamic forcing characteristics of dry cable galloping at critical Reynolds numbers. Eur J Mech - B/Fluids 49:243-249

Nikitas N, Macdonald JHG, Jakobsen JB (2012) Critical Reynolds number and galloping instabilities: experiments on circular cylinders. Exp Fluids 52:1295-1306

Norberg C (1993) Flow around rectangular cylinders: pressure forces and wake frequencies. J Wind Eng Ind Aerodyn 49:187-196

Obasaju ED (1983) An investigation of the effects of incidence on the flow around a square section cylinder. The Aeronaut Quart 34(4):243-259

Okajima A (1982) Strouhal numbers of rectangular cylinders. J Fluid Mech 123:379-398

van Oudheusden BW, Scarano F, van Hinsberg NP, Watt DW (2005) Phase-resolved characterization of vortex shedding in the near wake of a square-section cylinder at incidence. Exp Fluids 39:86-98

van Oudheusden BW, Scarano F, van Hinsberg NP, Roosenboom EWM (2007) Quantitative visualization of the flow around a square-section cylinder at incidence. J Wind Eng Ind Aerodyn 96:1-10

Ozgoren M (2006) Flow structure in the downstream of square and circular cylinders. Flow Measurement Instrum 17:225-235

Païdoussis MP, Price SJ, de Langre E (2011) Fluid-Structure Interaction. Cross-Flow-Induced Instabilities. Cambridge University Press, Cambridge

Povoa PHC, Granzoto RM, Becker GG, Lopez MR (2018) Landing gear drag reduction using lattice boltzmann method. 2018 AIAA Aerospace Sciences Meeting, Kissimmee, Florida, USA, AIAA 2018-1556

Ribeiro JLD (1991) Effects of surface roughness on the two-dimensional flow past circular cylinders I: mean forces and pressures. J Wind Eng Ind Aerodyn 37:299-309

Rockwell DO (1977) Organized fluctuations due to flow past a square cross section cylinder. J Fluids Eng 99(3):511-516

Roshko A (1961) Experiments on the flow past a circular cylinder at very high Reynolds number. J Fluid Mech 10:345-356 
Saha AK, Muralidhar K, Biswas G (2000) Experimental study of flow past a square cylinder at high Reynolds numbers. Exp in Fluids 29:553-563

Saha AK, Biswas G, Muralidhar K (2001) Two-dimensional study of the turbulent wake behind a square cylinder subject to uniform flow. J of Fluids Eng 123(3):595-603

Schewe G (1983) On the force fluctuations acting on a circular cylinder in crossflow from subcritical up to transcritical Reynolds numbers. J Fluid Mech 133:265-285

Shukla S, Sinha SS, Singh SN (2019) Ship-helo coupled airwake aerodynamics: a comprehensive review. Prog Aerosp Sci 106:71-107

Sohankar A (2006) Flow over a bluff body from moderate to high Reynolds numbers using large eddy simulation. Comput Fluids 35:1154-1168

Sohankar A, Mohagheghian S, Dehghan AA, Manshadi MD (2015) A smoke visualization study of the flow over a square cylinder at incidence and tandem square cylinders. J Vis 18:687-703

Tamura T, Miyagi T (1999) The effect of turbulence on aerodynamic forces on a square cylinder with various corner shapes. J Wind Eng Ind Aerodyn 83:135-145

Tanaka H (2003) Aerodynamics of cables. In: Proc. of the 5th International symposium on cable dynamics, Santa Margherita Ligure, Italy

Trias FX, Gorobets A, Oliva A (2015) Turbulent flow around a square cylinder at Reynolds number 22,000: a DNS study. Comput Fluids 123:87-98
Virlogeux M (2005) State-of-the-art in cable vibrations of cable-stayed bridges. Bridge Struct 1(3):133-168

Williamson CHK (1996) Vortex dynamics in the cylinder wake. Annual Review of Fluid Mechanics 28:477-539

Yamagishi Y, Oki M (2004) Effect of groove shape on flow characteristics around a circular cylinder with grooves. J Vis 7:209-16

Yen SC, Yang CW (2011) Flow patterns and vortex shedding behavior behind a square cylinder. J Wind Eng Ind Aerodyn 99:868-878

Yu Y, Kwok KCS, Liu XP, Zhang Y (2017) Air pollutant dispersion around high-rise buildings under different angles of wind incidence. J Wind Eng Ind Aerodyn 167:51-61

Zdravkovich MM (1997) Flow Around Circ Cylind. Oxford University Press, Oxford, UK

$\mathrm{Zu} \mathrm{G}, \mathrm{Lam}$ KM (2018) LES and wind tunnel test of flow around two tall buildings in staggered arrangement. Computation 6(2):1-18

Publisher's Note Springer Nature remains neutral with regard to jurisdictional claims in published maps and institutional affiliations. 